\title{
Effects of Zinc supplementation on serum lipids: a systematic review and meta-analysis
}

Priyanga Ranasinghe ${ }^{1 *}$, WS Wathurapatha ${ }^{1}$, MH Ishara ${ }^{2}$, R. Jayawardana ${ }^{3,4}$, P. Galappatthy ${ }^{1}$, P. Katulanda ${ }^{4}$ and GR Constantine ${ }^{4}$

\begin{abstract}
Zinc is a mineral that plays a vital role in many biological processes and plays an important role in insulin action and carbohydrate metabolism. It may also have a protective role in the prevention of atherogenesis. Numerous studies have evaluated the effects of Zinc supplementation on serum lipids in humans and have demonstrated varying results. We systematically evaluated the literature and performed a meta-analysis on the effects of Zinc supplementation on serum lipids. A five staged comprehensive search of the literature was conducted in the following databases; PubMed, Web of Science and SciVerse Scopus for studies published before 31st December 2014. All controlled clinical trial in humans, that included a Zinc supplement intervention, either alone or in combination with other micronutrients and evaluated effects on serum lipids (total cholesterol [TC], triglycerides [TG], LDL cholesterol [LDL-c] and HDL cholesterol [HDL-c]). A meta-analysis of selected studies was performed using RevMan v5.3. The Jaded scale was used to assess the methodological quality of the trials included in the systematic review. A total of 24 studies were included in Meta analysis, which included a total of 33 Zinc interventions, in a total of 14,515 participants in the Zinc intervention or control group. The duration of Zinc supplementation ranged from 1 month to 7.5 years. The dose of elemental Zinc supplemented ranged from 15-240 mg/day. The pooled mean difference for TC between Zinc supplemented and placebo groups from random effects analysis was $-10.92 \mathrm{mg} / \mathrm{dl}$ (95\% Cl: $-15.33,-6.52 ; \mathrm{p}<0.0001, \mathrm{I}^{2}=83 \%$ ), while for HDL cholesterol it was $2.12 \mathrm{mg} / \mathrm{dl}$ (95\% Cl: $-0.74,4.98 ; \mathrm{p}=0.15$, $\mathrm{I}^{2}=83 \%$ ). The pooled mean difference for $\mathrm{LDL}-\mathrm{c}$ between Zinc supplemented and placebo group from random effect analysis was $-6.87 \mathrm{mg} / \mathrm{dl}\left(95 \% \mathrm{Cl}:-11.16,-2.58 ; \mathrm{p}<0.001, \mathrm{I}^{2}=31\right)$ and for TG it was $-10.92 \mathrm{mg} / \mathrm{dl}(95 \% \mathrm{Cl}:-18.56,-3.28$; $\left.p<0.01, I^{2}=69 \%\right)$. In conclusion, Zinc supplementation has favourable effects on plasma lipid parameters. Zinc supplementation significantly reduced total cholesterol, LDL cholesterol and triglycerides. Therefore it may have the potential to reduce the incidence of atherosclerosis related morbidity and mortality.
\end{abstract}

\section{Introduction}

Zinc is a mineral that plays a vital role in many biological processes, such as enzyme action, cell membrane stabilization, gene expression and cell signaling [1]. It is required for structural and functional integrity of more than 2000 transcription factors and 300 enzymes; hence, almost all metabolic pathways are in some ways reliant on at least one Zinc requiring protein [2, 3]. Zinc also plays an important role in insulin action and carbohydrate metabolism [4]. Studies have shown that diabetes is

\footnotetext{
* Correspondence: priyanga.ranasinghe@gmail.com

'Department of Pharmacology, Faculty of Medicine, University of Colombo, Colombo, Sri Lanka

Full list of author information is available at the end of the article
}

accompanied by hypozincemia and hyperzincuria $[5,6]$. In addition Zinc is also an integral part of key anti-oxidant enzymes and Zinc deficiency impairs their synthesis, resulting in increased oxidative stress [7].

Zinc deficiency is known to affect $1 / 3^{\text {rd }}$ of the world's population [8]. It is estimated that Zinc deficiency is a major factor contributing to $1.4 \%$ of deaths worldwide [8]. Zinc deficiency is more common in developing countries, and although severe deficiency is rare in developed countries, marginal deficiency is thought to be relatively common $[9,10]$. Zinc deficiency is associated with many diseases, including malabsorption syndrome, chronic liver disease, chronic renal disease, sickle cell
Ciomed Central

(c) 2015 Ranasinghe et al. This is an Open Access article distributed under the terms of the Creative Commons Attribution License (http://creativecommons.org/licenses/by/4.0), which permits unrestricted use, distribution, and reproduction in any medium, provided the original work is properly credited. The Creative Commons Public Domain Dedication waiver (http:// creativecommons.org/publicdomain/zero/1.0/) applies to the data made available in this article, unless otherwise stated. 
disease, diabetes and malignancy [11]. Animal studies have shown that Zinc deficiency has profound effects on the cell structure of the aorta, fatty acid metabolism and carbohydrate metabolism, being disadvantageous for maintaining vascular health [12]. Zinc deficiency renders vascular endothelial cells more susceptible to the effects of oxidative stress [13, 14]. Furthermore, in LDL receptor knock-out mice acute Zinc deficiency elicits changes in key transcription factors and adhesion molecules that are pro-atherogenic [15]. In human studies a strong negative association was observed between the dietary intake of Zinc and the incidence of diabetes and heart disease, as well as several of their associated risk factors including hypertension and hyper-triglyceridemia [16]. Hence Zinc may have a protective role in the prevention of atherogenesis [12].

Several human studies have demonstrated that Zinc supplementation reduces total cholesterol, LDL cholesterol and triglycerides, in addition to increasing the HDL cholesterol levels [17-20]. However, these results have been contradicted by other studies [21-23]. Even under the most rigorous study design conditions, a single wellplanned study rarely provides definitive results [24]. Hence, changing clinical practices relying on a single high-profile clinical trial can be harmful to patients' health. Systematic reviews and meta-analyses on the other hand often have increased power and decreased bias as compared with the individual studies they include, and the careful pooling of treatment effects can provide the most accurate overall assessment of an intervention [24]. In 2008 Foster et al. performed a meta-analysis of controlled clinical trials to determine the effect of Zinc supplementation on serum lipids in humans [25]. They did not observe any beneficial effect of Zinc supplementation on plasma lipoproteins in the overall analysis, whilst in sub group analysis of healthy subjects Zinc supplementation was associated with a reduction in HDL cholesterol concentrations [25]. However, since then several recent studies have evaluated the effects of Zinc supplementation on serum lipids in humans and have demonstrated varying results [17, 21-23, 25-28]. Hence the present study aims to re-explore the area under discussion, by systematically evaluating the literature and performing an up to date meta-analysis on the effects of Zinc supplementation on serum lipids: total cholesterol (TC); LDL cholesterol (LDL-c); HDL cholesterol (HDL-c); and triglycerides (TG) in humans.

\section{Methods}

The current systematic review was conducted in accordance with the PRISMA statement (Preferred Reporting Items for Systematic reviews and Meta-Analyses) for systematic reviews of interventional studies [29].

\section{Literature search}

A five staged comprehensive search of the literature was conducted in the following databases; PubMed ${ }^{\circ}$ (U.S. National Library of Medicine, USA), Web of Science ${ }^{\circ}$ [v.5.4] (Thomson Reuters, USA) and SciVerse Scopus ${ }^{\circ}$ (Elsevier Properties S.A, USA) for studies published before $31^{\text {st }}$ December 2014. During the first stage the above databases were searched using the following keywords; ('Zinc' or 'Zn' or 'Zinc supplementation' or 'Zn supplementation' or 'Zinc therapy' or 'Zn therapy') AND ('Lipid(s)'or 'Cholesterol' or 'LDL' or 'HDL' or 'Lipoprotein(s)' or 'Triglyceride(s)').

In the second stage the total hits from the 3 databases were pooled and duplicates were removed. This was followed by screening of the retrieved articles by reading the article 'title' in the third stage and 'abstracts' in stage four. In the fifth stage individual manuscripts were screened, and those not satisfying inclusion criteria were excluded. To obtain additional data a manual search of the reference lists of articles selected in stage five was performed. This search process was conducted independently by two reviewers (PR and RJ) and the final group of articles to be included in the review was determined after an iterative consensus process.

\section{Inclusion and exclusion criteria}

A study was considered eligible for data extraction if it was a controlled clinical trial in humans, that included a Zinc supplement intervention, either alone or in combination with other micronutrients and evaluated at least one of the following outcomes: TG,TC, LDL-c and HDL-c. Results were limited to studies conducted in humans, published in English, while conference proceedings, editorials, commentaries and book chapters/book reviews were excluded.

\section{Data extraction and analysis}

A meta-analysis of selected studies examining the effects of Zinc supplementation on serum lipid parameters was performed using the Rev Man version 5.3 (Review Manager, Copenhagen: The Nordic Cochrane Centre, The Cochrane Collaboration, 2011) statistical software package. A random effect analysis was conducted for all comparisons and in all analyses a $p$-value $<0.05$ was considered statistically significant. Forest plots were used to illustrate the study findings and metaanalysis results. Statistical heterogeneity was assessed using the $x^{2}$ test on Cochrane's $Q$ statistic [30] and by calculating $I^{2}$ [31] and is considered significant if $\mathrm{p}<0.05$. TC, LDL-c, HDL-c and TG are reported as $\mathrm{mg} / \mathrm{dl}$, where studies reported as $\mathrm{mmol} / \mathrm{l}$ a numerical conversion to $\mathrm{mg} / \mathrm{dl}$ was done as follows; For TC, HDL-c, and LDL-c cholesterol, values presented in $\mathrm{mmol} / \mathrm{l}$ multiplied by conversion factor 38.67 and for TG, conversion factor used 
was 88.57 [32]. In a study where there was several interventions including interventions with multiple supplements, the interventions using Zinc supplementation alone was used in the meta analysis as the elemental dose of Zinc was similar in all interventions [33]. In a study done by Farvid et al. intervention with least number of additional supplements was used to compare with the placebo group [34]. In cross-over studies the pooled estimate of Zinc and placebo groups after completion of entire crossover scheme was used in the analysis [17, 19, 35]. Three separate sub-group analyses were performed; a) for group of studies using Zinc supplementation alone [17-19, 21-23, 26, 27, 33, 35-43], b) for group of studies done on healthy participants [35-37, 39-41, 44] and c) for group of studies done on non-healthy participants [17-23, 26-28, 33-35, 38, 42, 43, 45, 46].

\section{Quality assessment}

The Jaded scale was used to assess the methodological quality of the trials included in the systematic review [47]. Each study was scored from 0 ('poor' quality) to 5 ('good' quality) according to the following criteria: 1) was the study described as randomized? ;2) was the study described as double blind?; 3) was there a description of withdrawals and dropouts? 3) was the method of randomization described in the paper and appropriate?; 4) was the method of blinding described and appropriate?. Each question would score a single point if the answer is 'yes' or zero points if the answer is 'no'. Questions 4) and 5) would score -1 mark each if method of randomization was described, but inappropriate and method of blinding was described, but inappropriate respectively.

\section{Results}

\section{Literature search}

Literature search was done according to the above search criteria and the search strategy is summarized in Fig. 1. The number of articles identified initially from the different databases were as follows; PubMed $(\mathrm{n}=$ 995), Web of Science $(n=802)$, SciVerse Scopus $(n=$ 549). Five additional articles were identified by manual searching the reference lists of included studies. After removal of duplicates and screening of studies by reading the title, abstract and finally the full text, 32 studies were eligible to be included in systematic review. Descriptions of included studies are presented in Table 1.

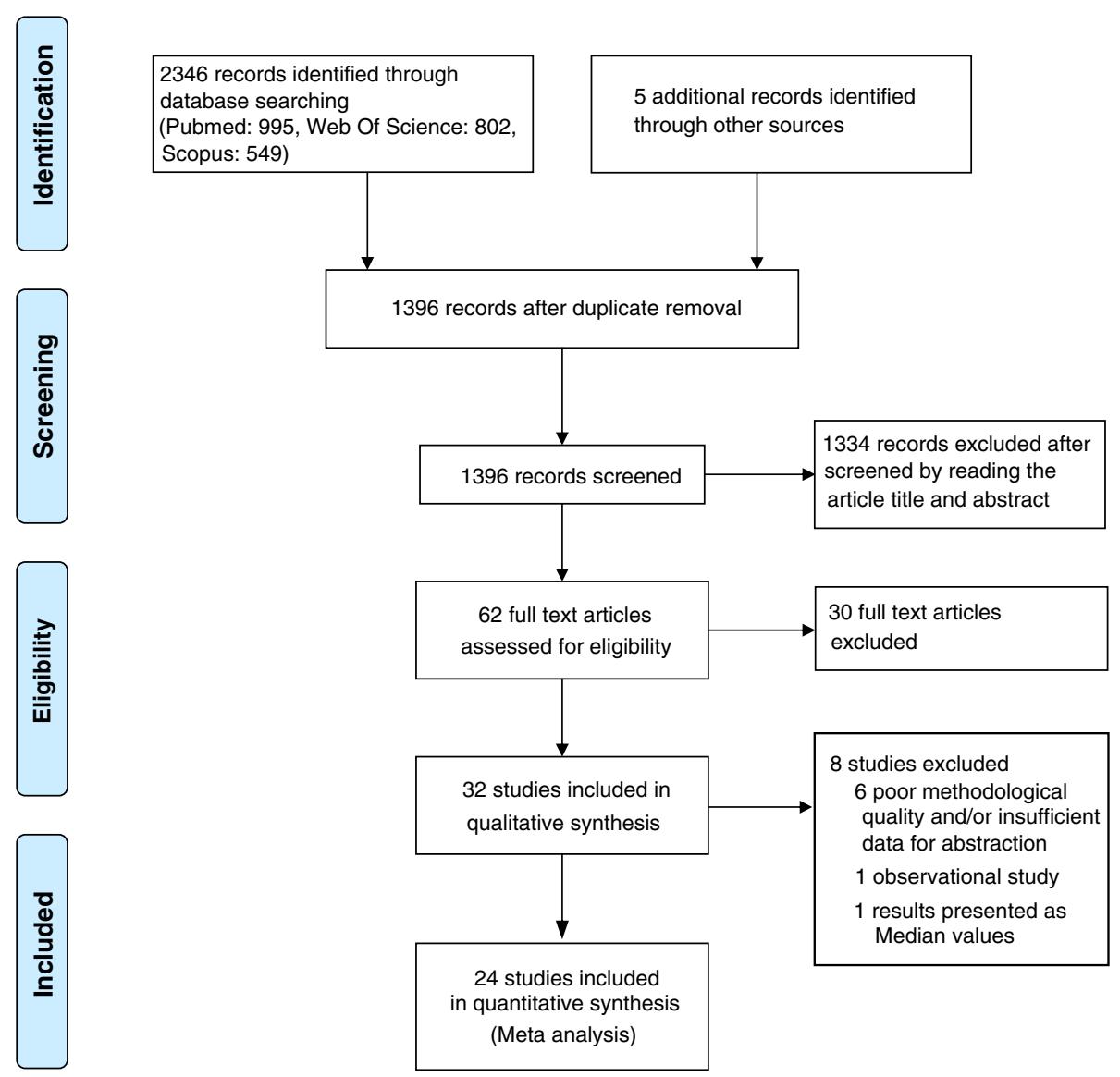

Fig. 1 Summarized search strategy 
Table 1 Description of included studies

\begin{tabular}{|c|c|c|c|c|c|c|c|c|c|}
\hline $\begin{array}{l}\text { Authors }{ }^{\text {[ref] }} \text { Year of } \\
\text { Publication; Country }\end{array}$ & $\begin{array}{l}\text { Study } \\
\text { design }\end{array}$ & $\begin{array}{l}\text { Duration of } \mathrm{Zn} \\
\text { supplementation }\end{array}$ & $\begin{array}{l}\text { n control(s)/placebo, } \\
\text { n Zn supplement(s) } \\
n \text { Other supplements }\end{array}$ & Gender & Age & Health status & $\begin{array}{l}\text { Formulation } \\
\text { Elemental Zn } \\
\text { dose(s) }\end{array}$ & $\begin{array}{l}\text { Lipid } \\
\text { parameters } \\
\text { studied }\end{array}$ & Significant outcomes \\
\hline $\begin{array}{l}\text { Afkhami-Ardekani et al. } \\
\text { [18] 2008; Iran }\end{array}$ & $R, P$ & 1.5 months & 2020 & Both & $\begin{array}{l}52.67 \pm \\
8.6\end{array}$ & $\begin{array}{l}\text { Type-2 diabetes } \\
\text { patients }\end{array}$ & $\begin{array}{l}\mathrm{ZnSO}_{4} 660 \mathrm{mg} / \\
\text { day }^{\mathrm{a}}\end{array}$ & $\begin{array}{l}\text { TG, TC, HDL-C, } \\
\text { LDL-C }\end{array}$ & Reduction in TG, TC and LDL \\
\hline $\begin{array}{l}\text { Age-Related Eye Disease } \\
\text { Study Research Group [48] } \\
\text { 2002; United States }\end{array}$ & $R, D B, P$ & 5 years & $\begin{array}{l}166202(\mathrm{Zn}), 168 \\
\text { (Zn + antioxidants) } \\
181 \text { (antioxidants) }\end{array}$ & Both & $55-80$ & $\begin{array}{l}\text { Patients with } \\
\text { Age-related } \\
\text { macular } \\
\text { degeneration }\end{array}$ & ZnO 80 mg/day & $\begin{array}{l}\text { TG, TC, HDL-C, } \\
\text { LDL-C }\end{array}$ & $\begin{array}{l}\text { Lipid profile not significantly } \\
\text { affected by long-term } \\
\text { supplementation with Zinc }\end{array}$ \\
\hline $\begin{array}{l}\text { Black et al. [36] 1988; } \\
\text { United States }\end{array}$ & $R, D B, P$ & 3 months & 913,9 & Males & $19-29$ & Healthy & $\begin{array}{l}\text { Zn gluconate } \\
50 \mathrm{mg} / \text { day } \\
(\mathrm{n}=13) 75 \mathrm{mg} / \text { day } \\
(\mathrm{n}=9)\end{array}$ & $\begin{array}{l}\text { TG, TC, HDL-C, } \\
\text { LDL-C, VDLL }\end{array}$ & $\begin{array}{l}\text { Serum TC, VLDL, LDL-C, TG } \\
\text { not affected. Both Zn groups } \\
\text { had significantly lower HDL } \\
\text { than placebo group and lower } \\
\text { than baseline }\end{array}$ \\
\hline $\begin{array}{l}\text { Bogden et al. [37] 1988; } \\
\text { United States }\end{array}$ & $R, D B, P$ & 3 months & 3636,31 & Both & $60-89$ & Healthy & $\begin{array}{l}\text { Zn acetate } \\
15 \mathrm{mg} / \text { day } \\
(\mathrm{n}=36) 100 \mathrm{mg} / \text { day } \\
(\mathrm{n}=31)\end{array}$ & TC, HLD-C, & $\begin{array}{l}\text { Serum TC and } \mathrm{HDL}-\mathrm{C} \text { was not } \\
\text { altered significantly by Zinc }\end{array}$ \\
\hline \multirow{3}{*}{$\begin{array}{l}\text { Boukaïba et al. [35] 1993; } \\
\text { France }\end{array}$} & \multirow[t]{3}{*}{$\mathrm{R}, \mathrm{DB}, \mathrm{C}$} & \multirow[t]{3}{*}{2 months } & 23,21 & \multirow[t]{3}{*}{ Both } & \multirow[t]{3}{*}{ 73-106 } & Healthy 2 groups & \multirow{3}{*}{$\begin{array}{l}\text { Zn gluconate } \\
20 \mathrm{mg} / \text { day }\end{array}$} & \multirow{3}{*}{$\begin{array}{l}\text { TG, TC, HDL-C, } \\
\text { LDL-C }\end{array}$} & \multirow{3}{*}{$\begin{array}{l}\text { Reduced TC in both Lean and } \\
\text { Reference groups than placebo. } \\
\text { Reduced HDL in Reference group. } \\
\text { Higher TG in Lean group. No effect } \\
\text { on TG in Reference group. Ratio of } \\
\text { LDL-c to HDL-c not affected. }\end{array}$} \\
\hline & & & \multirow[t]{2}{*}{23,21} & & & $\begin{array}{l}\text { Reterence- BMl } \\
>24 \mathrm{~kg} / \mathrm{m}^{2} \\
(\mathrm{n}=23)\end{array}$ & & & \\
\hline & & & & & & $\begin{array}{l}\text { Lean }-\mathrm{BMI}<21 \\
\mathrm{~kg} / \mathrm{m}^{2}(\mathrm{n}=21)\end{array}$ & & & \\
\hline \multirow{2}{*}{$\begin{array}{l}\text { Brewer et al. [49] 1991; } \\
\text { United States }\end{array}$} & \multirow[t]{2}{*}{$\mathrm{O}$} & \multirow[t]{2}{*}{$1-5$ years } & No controls & \multirow[t]{2}{*}{ Both } & \multirow[t]{2}{*}{ NM } & \multirow[t]{2}{*}{ Wilson's disease } & \multirow[t]{2}{*}{ NM } & $\mathrm{TG}, \mathrm{TC}, \mathrm{HDL}-\mathrm{C}$ & \multirow{2}{*}{$\begin{array}{l}\text { HDL level reduced in males only. } \\
\text { TC reduced in both genders. }\end{array}$} \\
\hline & & & $11(\mathrm{~F}), 13(\mathrm{M})$ & & & & & LDL-C, TC/HDL-C & \\
\hline \multirow{2}{*}{$\begin{array}{l}\text { Chevalier et al. [38] 2002; } \\
\text { United States }\end{array}$} & \multirow[t]{2}{*}{$R, D B, P$} & \multirow[t]{2}{*}{3 months } & 10 & \multirow[t]{2}{*}{ Both } & \multirow[t]{2}{*}{$23-80$} & End-Stage Renal & $\mathrm{ZnSO}_{4}$ & $\mathrm{TC}, \mathrm{HDL}-\mathrm{C}$ & \multirow{2}{*}{$\begin{array}{l}\text { TC and LDL-C increased. No } \\
\text { change in HDL-C }\end{array}$} \\
\hline & & & 10 & & & $\begin{array}{l}\text { Disease on } \\
\text { Haemodialysis }\end{array}$ & 50 mg/day & LDL-c & \\
\hline \multirow{5}{*}{$\begin{array}{l}\text { Crouse et al. [39] 1984; } \\
\text { United States }\end{array}$} & \multirow[t]{5}{*}{$R, D B, P$} & \multirow[t]{5}{*}{2 months } & 10,11 & \multirow[t]{5}{*}{ Males } & \multirow[t]{5}{*}{$20-55$} & Healthy & $\mathrm{ZnSO}_{4}$ & $\mathrm{TG}, \mathrm{TC}, \mathrm{HDL}-\mathrm{C}$ & \multirow{5}{*}{$\begin{array}{l}\text { No significant change in lipid } \\
\text { parameters in both groups }\end{array}$} \\
\hline & & & & & & 2 groups & $28.7 \mathrm{mg} /$ day & LDL-C & \\
\hline & & & 11,12 & & & $\begin{array}{l}\text { Endurance } \\
\text { trained }\end{array}$ & & & \\
\hline & & & & & & $(n=21)$ & & & \\
\hline & & & & & & Sedentary $(n=23)$ & & & \\
\hline \multirow[t]{2}{*}{ Farvid et al. [34] 2004; Iran } & \multirow[t]{2}{*}{$R, D B, P$} & \multirow[t]{2}{*}{3 months } & 18 & \multirow[t]{2}{*}{ Both } & \multirow[t]{2}{*}{$30-69$} & Type-2 & $\mathrm{ZnSO}_{4}$ & $\mathrm{TG}, \mathrm{TC}, \mathrm{HDL}-\mathrm{C}$ & Co-supplementation of $\mathrm{Mg}, \mathrm{Zn}$, \\
\hline & & & $\begin{array}{l}16(\mathrm{Zn}+\mathrm{Mg}), 17 \\
(\mathrm{Zn}+\mathrm{Mg}+\text { Vit.C + Vit.E) } \\
18 \text { (Vit C+ Vit.E) }\end{array}$ & & & Diabetes patients & 30 mg/day & LDL-C & $\begin{array}{l}\text { Vitamins } C \text { and } E \text { significantly } \\
\text { increases HDL-C. TG, TC, LDL-C } \\
\text { not altered }\end{array}$ \\
\hline Federico et al. [45] 2001; & $\mathrm{R}, \mathrm{CC}$ & 2 months & 30 & Both & $46-61$ & Gut cancer & NM & TC & No Significant reduction in TC \\
\hline
\end{tabular}


Table 1 Description of included studies (Continued)

\begin{tabular}{|c|c|c|c|c|c|c|c|c|c|}
\hline & & & $30(\mathrm{Zn}+\mathrm{Se})$ & & & & 21 mg/day & & \\
\hline \multirow[t]{3}{*}{$\begin{array}{l}\text { Feillet-Coudray et al. [40] } \\
\text { 2006; France }\end{array}$} & \multirow[t]{3}{*}{$R, D B, P$} & \multirow[t]{3}{*}{6 months } & $\begin{array}{l}16(\mathrm{~F}), 16(\mathrm{M}) \\
16(\mathrm{~F}), 16(\mathrm{M})\end{array}$ & \multirow[t]{3}{*}{ Both } & \multirow[t]{3}{*}{$55-70$} & \multirow[t]{3}{*}{ Healthy } & Zn gluconate & $\mathrm{TG}, \mathrm{TC}, \mathrm{HDL}-\mathrm{C}$ & \multirow[t]{3}{*}{$\begin{array}{l}\text { No significant change in lipid } \\
\text { parameters }\end{array}$} \\
\hline & & & \multirow{2}{*}{$\begin{array}{l}16(\mathrm{~F}), 16(\mathrm{M}), \\
15(\mathrm{~F}), 16(\mathrm{M})\end{array}$} & & & & $15 \mathrm{mg} / \operatorname{day}(\mathrm{n}=32)$ & \multirow[t]{2}{*}{$\mathrm{LDL}-\mathrm{C}$} & \\
\hline & & & & & & & $30 \mathrm{mg} / \operatorname{day}(\mathrm{n}=31)$ & & \\
\hline \multirow{3}{*}{$\begin{array}{l}\text { Foster et al. [33] 2013; } \\
\text { Australia }\end{array}$} & \multirow[t]{3}{*}{$R, D B, P$} & \multirow[t]{3}{*}{3 months } & 10 & \multirow[t]{3}{*}{ Females } & \multirow{3}{*}{$\begin{array}{l}65.0 \pm \\
7.8\end{array}$} & Type-2 & Zn sulphate & $\mathrm{TG}, \mathrm{TC}, \mathrm{HDL}-\mathrm{C}$ & \multirow{3}{*}{$\begin{array}{l}\text { No significant change in lipid } \\
\text { parameters }\end{array}$} \\
\hline & & & $12(Z n), 11(Z n+A L A)$ & & & Diabetes patients & 40 mg/day & LDL-C & \\
\hline & & & $10(\mathrm{ALA})$ & & & & & & \\
\hline \multirow{4}{*}{$\begin{array}{l}\text { Freeland-Graves et al. [50] } \\
\text { 1982; United States }\end{array}$} & \multirow[t]{4}{*}{$R, D B, P$} & \multirow[t]{4}{*}{2 months } & 8 & \multirow[t]{4}{*}{ Females } & \multirow[t]{4}{*}{$18-40$} & \multirow[t]{4}{*}{ Healthy } & Zn Acetate & $\mathrm{HDL}-\mathrm{C}$ & \multirow{4}{*}{$\begin{array}{l}\text { A transient non-dose related } \\
\text { reduction in } \mathrm{HDL}-\mathrm{c}\end{array}$} \\
\hline & & & & & & & 15 mg/day $(n=8)$ & & \\
\hline & & & $8,8,8$ & & & & 50 mg/day $(n=8)$ & & \\
\hline & & & & & & & 100 mg/day $(n=8)$ & & \\
\hline \multirow{3}{*}{$\begin{array}{l}\text { Gatto and Samman [51] } \\
\text { 1995; Australia }\end{array}$} & \multirow[t]{3}{*}{$R, B, C$} & \multirow[t]{3}{*}{1 month } & 10 & \multirow[t]{3}{*}{ Males } & \multirow{3}{*}{$\begin{array}{l}24.3 \pm \\
4.2\end{array}$} & \multirow[t]{3}{*}{ Healthy } & $\mathrm{ZnSO}_{4}$ & $\mathrm{TG}, \mathrm{TC}$ & \multirow{3}{*}{$\begin{array}{l}\text { No significant changes in TC, } \\
\mathrm{LDL} \text {-c or TG. } \mathrm{HDL}_{2}: \mathrm{HDL}_{3} \text { ratio } \\
\text { increased }\end{array}$} \\
\hline & & & 10 & & & & $50 \mathrm{mg} /$ day & HDL-c, & \\
\hline & & & & & & & & $\mathrm{LDL}-\mathrm{C}$ & \\
\hline $\begin{array}{l}\text { Gunasekara et al. [46] 2011; } \\
\text { Sri Lanka }\end{array}$ & $R, B, P$ & 4 months & 32 & Both & $\begin{array}{l}M-54.6 \\
\pm 7.0\end{array}$ & Type-2 & $\mathrm{ZnSO} 4$ & $\mathrm{TG}, \mathrm{TC}, \mathrm{HDL}-\mathrm{C}$ & $\begin{array}{l}\text { Reduced TC, LDL and TC/HDL-C } \\
\text { ratio only in the group receiving }\end{array}$ \\
\hline & & & $28(\mathrm{Zn}+\mathrm{MVM})$ & & F- 54.9 & Diabetes patients & 22 mg/day & LDL-C & Zinc + MVM supplementation. \\
\hline & & & 26 (MVM) & & & & & & \\
\hline Hashemipour et al. & $R, D B, C$ & 2 months & 60 & Both & $6-10$ & Obese & $\mathrm{ZnSO} 4$ & $\mathrm{TG}, \mathrm{TC}, \mathrm{HDL}-\mathrm{C}$ & TC, LDL-C and TG reduced. No \\
\hline & & & 60 & & & & 20 mg/day & $L D L-C$ & \\
\hline Hercberg et al. [44] & $R, D B, P$ & 7.5 years & $3869(F), 2508(M)$ & Both & $35-60$ & Healthy & Multi-vitamin & $\mathrm{TG}, \mathrm{TC}, \mathrm{HDL}-\mathrm{C}$ & Significant reduction of HDL-C \\
\hline & & & $3844(F), 2520(M)$ & & & & (Zn 20 mg/day) & LDL-C & in other lipid parameters \\
\hline Hininger-Favier et al. [41] & $R, D B, P$ & 6 months & 130 & Both & $55-85$ & Healthy & Zn Gluconate & $\mathrm{TG}, \mathrm{TC}, \mathrm{HDL}-\mathrm{C}$ & No effect with Zinc 15 mg/day \\
\hline & & & & & & & 15 mg/day & LDL-C, LDL:HDL & or 30 mg/day \\
\hline & & & 126,131 & & & & $(n=126)$ & & \\
\hline & & & & & & & 30 mg/day $(n=131)$ & & \\
\hline Hooper et al. [52] 1980, & $P$ & 5 weeks & 8 & Males & $23-35$ & Healthy & $\mathrm{ZnSO} 4$ & $\mathrm{TG}, \mathrm{TC}, \mathrm{HDL}-\mathrm{C}$ & $25 \%$ reduction of HDL-c. TC, TG, \\
\hline United States & & & 12 & & & & 160 mg/day & LDL-C & and LDL-c no signiticant change. \\
\hline $\begin{array}{l}\text { Kadhim et al. [20] 2006; } \\
\text { Iraq }\end{array}$ & $\mathrm{DB}, \mathrm{P}$ & 3 months & $\begin{array}{l}15 \text { (Metformin + } \\
\text { Placebo) }\end{array}$ & Both & $40-64$ & $\begin{array}{l}\text { Type-2 Diabetes } \\
\text { patients }\end{array}$ & Zn acetate & $\mathrm{TG}, \mathrm{TC}, \mathrm{HDL}-\mathrm{C}$ & $\begin{array}{l}\text { Reduced TC, TG and LDL-C. } \\
\text { HDL-c significantly increased. }\end{array}$ \\
\hline & & & $\begin{array}{l}18(\text { Metformin }+ \\
\text { Melatonin }+Z n)\end{array}$ & & & & $50 \mathrm{mg} /$ day $^{\mathrm{a}}$ & LDL-C & \\
\hline & & & 13 (Melatonin + Zn) & & & & & & \\
\hline
\end{tabular}


Table 1 Description of included studies (Continued)

\begin{tabular}{|c|c|c|c|c|c|c|c|c|c|}
\hline \multirow[t]{2}{*}{ Khan et al. [42], 2013; India } & \multirow[t]{2}{*}{$R, P$} & \multirow[t]{2}{*}{3 months } & 21 & \multirow[t]{2}{*}{ Both } & \multirow[t]{2}{*}{$40-69$} & \multirow{2}{*}{$\begin{array}{l}\text { Type-2 Diabetes } \\
\text { patients }\end{array}$} & $\mathrm{ZnSO4}$ & \multirow{2}{*}{$\begin{array}{l}\mathrm{TG}, \mathrm{TC}, \mathrm{HDL}-\mathrm{C}, \\
\mathrm{LDL}-\mathrm{C}\end{array}$} & \multirow{2}{*}{$\begin{array}{l}\text { Decrease in TG. HDL-C increased } \\
\text { No change in TC and LDL-C. }\end{array}$} \\
\hline & & & 23 & & & & 50 mg/day & & \\
\hline \multirow{2}{*}{$\begin{array}{l}\text { Kim and Lee [21] 2012; } \\
\text { South Korea }\end{array}$} & \multirow[t]{2}{*}{$P$} & \multirow[t]{2}{*}{2 months } & 20 & \multirow[t]{2}{*}{ Females } & \multirow[t]{2}{*}{$19-28$} & \multirow[t]{2}{*}{ Obese } & Zn gluconate & \multirow[t]{2}{*}{$\mathrm{TG}, \mathrm{TC}, \mathrm{HDL}-\mathrm{C}$} & \multirow{2}{*}{$\begin{array}{l}\text { No significant change in lipid } \\
\text { parameters }\end{array}$} \\
\hline & & & 20 & & & & 30 mg/day & & \\
\hline \multirow[t]{3}{*}{ Li et al. [28] 2010; China } & \multirow[t]{3}{*}{$R, D B, P$} & \multirow[t]{3}{*}{6.5 months } & 29 & \multirow[t]{3}{*}{ Females } & \multirow[t]{3}{*}{$18-55$} & \multirow[t]{3}{*}{ Obese } & Multi-vitamin & $\mathrm{TG}, \mathrm{TC}, \mathrm{HDL}-\mathrm{C}$ & \multirow{3}{*}{$\begin{array}{l}\text { Reduced TC and LDL-C and } \\
\text { increased HDL-C in multivitamin } \\
\text { \& mineral supplemented group. } \\
\text { No change in TG. }\end{array}$} \\
\hline & & & $\begin{array}{l}30 \text { (multivitamin \& } \\
\text { mineral) }\end{array}$ & & & & (Zn 15 mg/day) & LDL-C & \\
\hline & & & 28 (Calcium) & & & & & & \\
\hline \multirow{2}{*}{$\begin{array}{l}\text { Partida-Hernández et al. } \\
\text { [19] 2006, Mexico }\end{array}$} & \multirow[t]{2}{*}{$\mathrm{R}, \mathrm{DB}, \mathrm{C}$} & \multirow[t]{2}{*}{3 months } & 27 & \multirow[t]{2}{*}{ Males } & \multirow[t]{2}{*}{$35-65$} & Type-2 & $\mathrm{ZnSO} 4$ & $\mathrm{TG}, \mathrm{TC}, \mathrm{HDL}-\mathrm{C}$ & \multirow{2}{*}{$\begin{array}{l}\text { Reduced TC, TG, VLDL-c. } \\
\text { Increased } \mathrm{HDL}-\mathrm{C}\end{array}$} \\
\hline & & & 27 & & & Diabetes patients & $100 \mathrm{mg} /$ day $^{\mathrm{a}}$ & LDL-C, VLDL-C & \\
\hline \multirow{2}{*}{$\begin{array}{l}\text { Payahoo et al. [26] 2013; } \\
\text { Iran }\end{array}$} & \multirow[t]{2}{*}{$R, D B, P$} & 1 month & 30 & Both & $18-45$ & Obese & Zn gluconate & $\mathrm{TG}, \mathrm{TC}, \mathrm{HDL}-\mathrm{C}$ & TG level decreased. \\
\hline & & & 30 & & & & $30 \mathrm{mg} /$ day $^{\mathrm{a}}$ & LDL-C & $\begin{array}{l}\text { No change in other lipid } \\
\text { parameters }\end{array}$ \\
\hline Rahimi-Ardabili et al. [27] & $R, D B, P$ & 2 months & 30 & Both & $52.8 \pm$ & End-Stage Renal & $\mathrm{ZnSO} 4$ & $\mathrm{TG}, \mathrm{TC}, \mathrm{HDL}-\mathrm{C}$ & No change in TC, TG and LDL-C. \\
\hline 2012; Iran & & & 30 & & 12.7 & $\begin{array}{l}\text { Disease on } \\
\text { Haemodialysis }\end{array}$ & $100 \mathrm{mg} /$ day $^{\mathrm{a}}$ & LDL-C & Increase in $\mathrm{HDL}$ \\
\hline RangaRao et al. [53] 1990; & $\mathrm{CC}$ & 1 month & 5 & Males & NM & Type-1 & $\mathrm{ZnSO} 4$ & $\mathrm{TG}, \mathrm{TC}, \mathrm{HDL}-\mathrm{C}$ & No significant change in lipid \\
\hline & & & 7 & & & Diabetes patients & $660 \mathrm{mg} /$ day $^{\mathrm{a}}$ & & parameters \\
\hline Roozbeh et al. [22] 2009; & $R, D B, P$ & 1.5 months & 26 & Both & 55.7 & End-Stage Renal & $\mathrm{ZnSO} 4$ & $\mathrm{TG}, \mathrm{TC}, \mathrm{HDL}-\mathrm{C}$ & Increase in TC, TG, LDL-C and \\
\hline & & & 27 & & & $\begin{array}{l}\text { Disease on } \\
\text { Haemodialysis }\end{array}$ & $50 \mathrm{mg} /$ day & LDL-C & \\
\hline $\begin{array}{l}\text { Samman and Roberts [54] } \\
\text { 1988; Australia }\end{array}$ & $R, D B, C$ & 1.5 months & 41 & Both & $\begin{array}{l}M-28.2 \\
\pm 2.0\end{array}$ & Healthy & $\mathrm{ZnSO} 4$ & TC, LDL- & $\begin{array}{l}\text { No change in TC. In females LDL-C } \\
\text { reduced. HDL2 increased and }\end{array}$ \\
\hline & & & 41 & & F- 26.8 & & $150 \mathrm{mg} /$ day & $\mathrm{C}, \mathrm{HDL}-\mathrm{C}$ & HDL 3 decreased. \\
\hline & & & & & \pm 1.6 & & & HDL2, HDL3 & \\
\hline Seet et al. [23] 2011; & $R, B, P$ & 3 months & 20 & Males & NM & Type-2 & Zn gluconate & $\mathrm{TG}, \mathrm{TC}, \mathrm{HDL}-\mathrm{C}$ & No significant change in lipid \\
\hline & & & 20 & & & Diabetes patients & $240 \mathrm{mg} /$ day & $L D L-C$ & parameters \\
\hline Shah et al. [43] 1988; India & $R, P$ & 1 month & 10 & Males & $31-70$ & Ischaemic Heart & $\mathrm{ZnSO} 4$ & $\mathrm{TC}, \mathrm{TG}, \mathrm{a}-$ and & Significant reduction in TC, \\
\hline & & & 10 & & & Disease patients & $600 \mathrm{mg} /$ day $^{\mathrm{a}}$ & $\beta$-lipoprotein & $\begin{array}{l}\text { 3-lipoproteln. Increase In } \\
\text { a-lipoprotein. No change } \\
\text { in TG }\end{array}$ \\
\hline Thurnham et al. [55] 1988; & $R, D B, P$ & 13.5 months & $610^{b}$ & Both & $35-64$ & Healthy & Zn gluconate & TC & No significant change \\
\hline Cnilna & & & & & & & $50 \mathrm{mg} / \mathrm{d}$ & & \\
\hline
\end{tabular}

ALA-a-linolenic acid, B-Single blinded, C-Cross over; CC-Case-control, DB-Double Blinded, F-female, HDL-c-High Density Lipoprotein-Cholesterol, LDL-c-Low Density Lipoprotein-Cholesterol, $M$-male, MVM- multivitamin/ mineral,NM-Not Mentioned, $O$ - Observational, $P$-Parallel, $R$-Randomized, TC-Total Cholesterol, TG-Triglycerides, Age presented as mean \pm SD in years where data were available and as age range in other studies

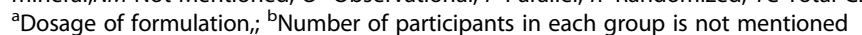


Only 24 studies were included in Meta analysis and the reasons for exclusion of 8 studies [48-55] are also mentioned in Fig. 1.

\section{Description of the studies and Quality assessment}

Studies that were included in the meta-analysis are English-language, human, controlled trials. Out of the total of 33 Zinc interventions included in meta-analysis, 26 interventions [17-19, 21-23, 26, 27, 33, 35-43] investigated the effects of Zinc supplementation alone on plasma lipids while other 7 interventions investigated the effect of supplementation of Zinc together with other vitamins and/or minerals. Duration of Zinc supplementation ranged from 1 month to 6.5 months with the exception of one long term study in which Zinc was supplemented for 7.5 years [44]. The dose of elemental Zinc supplemented in these interventions ranged from 15-240 mg/day (average dose of elemental Zinc per intervention: $39.3 \mathrm{mg} /$ day). A variety of Zinc anions were used, including sulfate $[17-19,22,27,33,34,38$, $39,42,43,46]$, gluconate $[21,23,26,35,36,40,41]$ and acetate $[20,37]$ or undefined $[28,44,45]$.

In total, 14,515 participants were assigned to a Zinc intervention or control group. The age range of participants was 19-106 years except one study which was done in children aged 6-10 years. Out of 24 studies, 7 studies (16 interventions) involved healthy participants. Of the remaining trials, 8 studies were undertaken in those with type 2 Diabetes, 4 studies in obese individuals, 3 studies in subjects with end stage renal failure undergoing haemodialysis, 1 study in gut cancer patients and 1 study in patients with Ischemic heart disease. The mean jaded scale score for all trials included in meta-analysis was 3.13 , out of a maximum score of 5 and 16 out of 24 studies scored $\geq 3$ marks (Table 2). Two studies that scored zero points were excluded from the meta-analysis due to poor methodological quality (Fig. 1) [49, 52].

\section{Effect Zinc supplementation on total cholesterol}

Effect of Zinc supplementation on total cholesterol concentration was studied in all 24 studies (33 interventions, $\mathrm{n}=14515)[17-23,26-28,33-46]$ included in the meta-analysis. There was a statistically significant reduction in TC concentration in the Zinc supplemented group. The pooled mean difference for TC between Zinc supplemented and placebo groups from random effects analysis was $-10.92 \mathrm{mg} / \mathrm{dl}$ (95 \% CI: $-15.33,-6.52 ; \mathrm{p}<0.0001)$. However statistical heterogeneity as indicated by $\mathrm{I}^{2}=83 \%$ $(\mathrm{p}<0.05)$ of the data prevents the evaluation of a pooled estimate for TC (Fig. 2 (I)). In the subgroup-analyses, the group of interventions (26 interventions, $\mathrm{n}=1528$ ) in which Zinc was supplemented alone demonstrated a similar, statistically significant reduction in TC concentration in comparison to control groups. The pooled mean difference for TC between Zinc supplemented and placebo groups from random effect analysis was $-10.72 \mathrm{mg} / \mathrm{dl}$ (95 \% CI: -19.01, -1.32; p <0.05) (Fig. 3(I)) and statistical heterogeneity as indicated by $\mathrm{I}^{2}=80 \%(\mathrm{p}<0.05)$. When studies were grouped by health status, reduction in TC in comparison to control groups was statistically significant and was greater in magnitude $(-17.02 \mathrm{mg} / \mathrm{dl}$ [95 \% CI: -30.52, -3.52; p < 0.05], $\left.\left[\mathrm{I}^{2}=87, \mathrm{p}<0.05\right]\right)$ among non-healthy participants (18 interventions, $\mathrm{n}=$ 866) (Fig. 4 (I)). Zinc supplementation among healthy participants (15 interventions, $\mathrm{n}=13,650)$ demonstrated minor but statistically significant reduction in TC $(-1.22 \mathrm{mg} / \mathrm{dl}$ [95 \% CI: $-2.17,-0.26 ; \mathrm{p}<0.05],\left[\mathrm{I}^{2}=\right.$ $0, \mathrm{p}>0.05]$ ) (Fig. 5(I)).

\section{Effect of Zinc supplementation on HDL cholesterol}

Twenty one studies (29 interventions, $\mathrm{n}=1,694$ ) [17-23, 26-28, 33-42, 46] explored the effect of Zinc supplementation on HDL cholesterol. The forest plot for overall analysis of HDL cholesterol (Fig. 2(II)) shows the pooled mean difference for HDL cholesterol between Zinc supplemented and placebo groups from random effect analysis was $2.12 \mathrm{mg} / \mathrm{dl}$ (95 \% CI: $-0.74,4.98$; p = $0.15)$ and statistical heterogeneity as indicated by $\mathrm{I}^{2}=83 \%$ $(\mathrm{p}<0.05)$. Also the group of interventions $(25$ interventions, $\mathrm{n}=1,508)$ employing Zinc supplementation alone did not demonstrate a statistically significant increase in HDL-c levels (+0.73 mg/dl, $95 \%$ CI: $-2.30,3.75, \mathrm{p}=0.64)$ in subgroup-analysis (Fig. 3(II)). However, as Fig. 4(II) illustrates Zinc supplementation among non-healthy participants (16 interventions, $\mathrm{n}=785$ ) demonstrated a considerable and statistically significant increase in HDL-c (+6.15 mg/dl [95 \% CI: 2.38, 9.92; $\mathrm{p}<0.05],\left[\mathrm{I}^{2}=85, \mathrm{p}<\right.$ $0.05])$. In contrast to this Zinc supplementation in healthy participants (13 interventions, $\mathrm{n}=909$ ) demonstrated a significant reduction in HDL-c $(-3 \mathrm{mg} / \mathrm{dl}$ [95 \% CI: -5.91 , 0.10; $\left.\mathrm{p}<0.05],\left[\mathrm{I}^{2}=45, \mathrm{p}<0.05\right]\right)$ (Fig. 5 (II)).

\section{Effect of Zinc supplementation on LDL cholesterol}

There were 17 studies $[17-20,22,23,26-28,33-35$, $39-42,46]$ (23 interventions, $\mathrm{n}=1,455)$ in which the effect of Zinc supplementation on LDL cholesterol was studied. Forest plot for LDL-c (Fig. 2(III)) shows there is a statistically significant reduction in LDL-c in Zinc supplemented group. The pooled mean difference for LDL-c between Zinc supplemented and placebo group from random effect analysis was $-6.87 \mathrm{mg} /$ dl (95 \% CI: $-11.16,-2.58 ; \mathrm{p}<0.001)$ and the statistical heterogeneity of the data as indicated by $\mathrm{I}^{2}=31$ was insignificant $(\mathrm{p}=0.08)$. Forest plot for subgroup analysis (Fig. 3(III)) of LDL-c shows the pooled mean difference for LDL-c between Zinc alone supplemented group and placebo groups from random effect analysis 
Table 2 Jaded scale

\begin{tabular}{|c|c|c|c|c|c|c|}
\hline Study & Randomised & $\begin{array}{l}\text { Double } \\
\text { blind }\end{array}$ & $\begin{array}{l}\text { Withdrawals and } \\
\text { drop outs }\end{array}$ & $\begin{array}{l}\text { Randomisationmethod } \\
\text { described and appropriate }\end{array}$ & $\begin{array}{l}\text { Blinding method described } \\
\text { and appropriate }\end{array}$ & Total \\
\hline Afkhami-Ardekani et al., 2008 & 1 & 0 & 1 & 0 & N/A & 2 \\
\hline $\begin{array}{l}\text { Age-Related Eye Disease Study Research } \\
\text { Group, } 2002\end{array}$ & 1 & 1 & 0 & 0 & 0 & 2 \\
\hline Black et al., 1988 & 1 & 1 & 1 & 0 & 0 & 3 \\
\hline Bogden et al., 1988 & 1 & 1 & 0 & 1 & 0 & 3 \\
\hline Boukaïba et al., 1993 & 1 & 1 & 1 & 0 & 0 & 3 \\
\hline Brewer et al., 1991 & 0 & 0 & 0 & 0 & 0 & 0 \\
\hline Chevalier et al., 2002 & 1 & 1 & 1 & 0 & 0 & 3 \\
\hline Crouse et al., 1984 & 1 & 1 & 1 & 0 & 0 & 3 \\
\hline Farvid et al., 2004 & 1 & 1 & 1 & 1 & 0 & 4 \\
\hline Federico at al., 2001 & 1 & 0 & 1 & 0 & N/A & 2 \\
\hline Feillet-Coudray et al., 2006 & 1 & 1 & 1 & 0 & 0 & 3 \\
\hline Foster et al., 2013 & 1 & 1 & 1 & 1 & 1 & 5 \\
\hline Freeland-Graves et al., 1982 & 1 & 1 & 0 & 0 & 0 & 2 \\
\hline Gatto et al., 1995 & 1 & 0 & 1 & 0 & N/A & 2 \\
\hline Gunasekara et al., 2011 & 1 & 0 & 1 & 1 & 0 & 3 \\
\hline Hashemipour et al., 2009 & 1 & 1 & 1 & 1 & 0 & 4 \\
\hline Hercberg et al., 2005 & 1 & 1 & 1 & 1 & 1 & 5 \\
\hline Hininger-Favier et al., 2007 & 1 & 1 & 1 & 1 & 1 & 5 \\
\hline Hooper et al., 1980 & 0 & 0 & 0 & 0 & 0 & 0 \\
\hline Kadhim et al., 2006 & 0 & 1 & 1 & N/A & 0 & 2 \\
\hline Khan et al., 2013 & 1 & 0 & 1 & 0 & N/A & 2 \\
\hline Kim et al., 2012 & 0 & 0 & 1 & 0 & 0 & 1 \\
\hline Li et al., 2010 & 1 & 1 & 1 & 1 & 0 & 4 \\
\hline Partida-Hernández et al., 2006 & 1 & 1 & 1 & 0 & 0 & 3 \\
\hline Payahoo et al., 2013 & 1 & 1 & 1 & 1 & 1 & 5 \\
\hline Rahimi-Ardabili et al., 2012 & 1 & 1 & 0 & 0 & 0 & 2 \\
\hline RangaRao et al., 1990 & 0 & 0 & 1 & 0 & 0 & 1 \\
\hline Roozbeh et al., 2009 & 1 & 1 & 1 & 0 & 1 & 4 \\
\hline Samman et al., 1988 & 1 & 1 & 1 & 0 & 0 & 3 \\
\hline Seet et al., 2011 & 1 & 0 & 1 & 0 & N/A & 2 \\
\hline Shah et al., 1988 & 1 & 0 & 1 & 0 & N/A & 2 \\
\hline Thurnham et al., 1988 & 1 & 1 & 0 & 0 & 0 & 2 \\
\hline
\end{tabular}

was $-4.78 \mathrm{mg} / \mathrm{dl}(95 \% \mathrm{CI}:-9.14,-0.43 ; \mathrm{p}<0.05)$ and the statistically heterogeneity was $\mathrm{I}^{2}=24(\mathrm{p}=0.17)$. When the interventions done in non- healthy participants (14 interventions, $n=725$ ) were grouped together, Zinc supplementation demonstrated a significant reduction in LDL-c $(-11.25 \mathrm{mg} / \mathrm{dl}$ [95 \% CI: -16.06, -6.44; $\mathrm{p}<0.05],\left[\mathrm{I}^{2}=15\right.$, $\mathrm{p}>0.05])$ and the magnitude of reduction was greater than that in overall ungrouped analysis (Fig. 4 (III)). In contrast to this, Zinc supplementation in healthy participants ( 9 interventions, $\mathrm{n}=730$ ) demonstrated a smaller and insignificant increase in LDL-c $(+1.12 \mathrm{mg} / \mathrm{dl}$ [95 \% CI: -3.93, 6.16; p > 0.05], $\left[\mathrm{I}^{2}=0, \mathrm{p}>0.05\right]$ ) (Fig. 5 (III)).

\section{Effect of Zinc supplementation on Triglycerides}

Effect of Zinc supplementation on Triglyceride concentration was studied in 19 studies (25 interventions, $\mathrm{n}=$ 1,503)[17-23, 26-28, 33-35, 39-43, 46] included in meta analysis. There was a statistically significant reduction in triglyceride concentration in Zinc supplemented group. The pooled mean difference for triglyceride between Zinc supplemented and placebo groups from random effects analysis was $-10.92 \mathrm{mg} / \mathrm{dl}$ (95 \% CI: $-18.56,-3.28$; p < $0.01)$ in the presence of statistical heterogeneity of the data as indicated by $\mathrm{I}^{2}=69 \%(\mathrm{p}<0.0001)$ (Fig. 2(IV)). Also subanalysis of the group of interventions in which Zinc was 


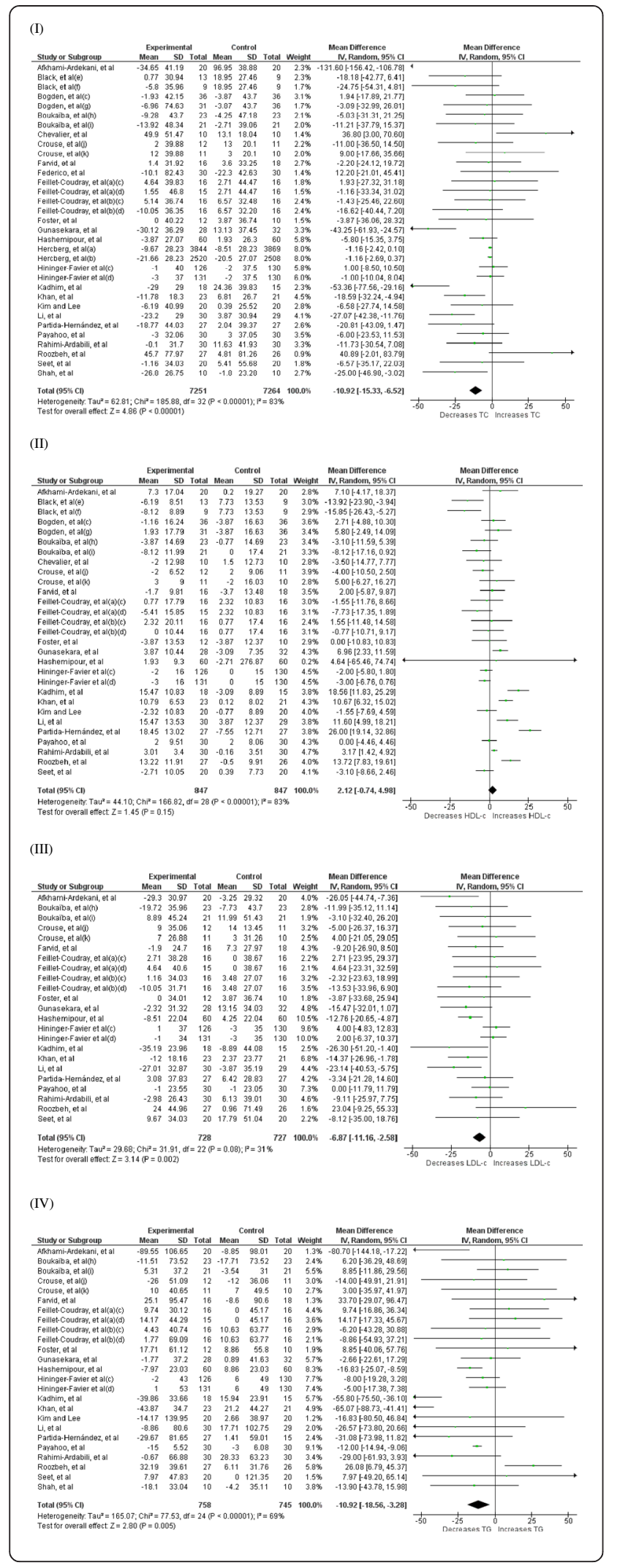

Fig. 2 Forest plots showing effect of Zinc supplementation on; (I) Total cholesterol, (II) HDL cholesterol, (III) LDL cholesterol, (IV) Triglycerides. a- female, (b)- male, (c)- Zinc supplementation 15 mg/day, (d)- Zinc supplementation 30 mg/day, (e)- Zinc supplementation $50 \mathrm{mg} /$ day, (f)- Zinc supplementation $75 \mathrm{mg} /$ day, (g)- Zinc supplementation $100 \mathrm{mg} /$ day, (h)- reference group, (i)- Lean group, (j)- Sedentary males, (k)- Trained males

supplemented alone (21 interventions, $\mathrm{n}=1,317$ ) demonstrated statistically significant reduction in TG levels in Zinc supplemented groups in comparison to their controls $(-8.73 \mathrm{mg} / \mathrm{dl}, 95 \% \mathrm{CI}:-16.29,-1.17, \mathrm{p}<0.05)$ and statistical heterogeneity as indicated by $\mathrm{I}^{2}=64 \%(\mathrm{p}<0.0001)$ (Fig. 3 (IV)). As Fig. 4 (IV) illustrates Zinc supplementation in non-healthy participants (16 interventions, $\mathrm{n}=773$ ) demonstrated a significant reduction in TG levels which was greater in magnitude than that in ungrouped analysis $(-17.59 \mathrm{mg} / \mathrm{dl}$ [95 \% CI: $-28.80,-6.39 ; \mathrm{p}<0.05],\left[\mathrm{I}^{2}=77\right.$, $\mathrm{p}$ $<0.05]$ ). However, Zinc supplementation in healthy participants (9 interventions, $\mathrm{n}=730$ ) did not demonstrate a significant reduction in TG levels $(-2.97 \mathrm{mg} / \mathrm{dl}[95 \%$ CI: -9.75, 3.81; p > 0.05], $\left[\mathrm{I}^{2}=0\right.$, p > 0.05]) (Fig. 5 (IV)).

\section{Other significant effects}

Gunasekara, et al. reported a significant reduction in Total cholesterol/HDL ratio from 3.39 to 3.21 ( $\mathrm{p}<0.05$ ) after Zinc supplementation [46]. Although not statistically significant, a study carried out by Brewer, et al. also reported reduction of this ratio after $\mathrm{Zn}$ supplementation in newly diagnosed female patients with Wilson's disease and patients who had received anti copper therapy (both genders) [49]. Zinc supplementation has shown significant reduction in VLDL cholesterol concentration in few studies $[19,36]$. Studies have shown Zinc supplementation results in cholesterol to shift from $\mathrm{HDL}_{3}$ to $\mathrm{HDL}_{2}$ causing an increase in $\mathrm{HDL}_{2} / \mathrm{HDL}_{3}$ ratio $[51,54]$.

\section{Discussion}

This comprehensive systematic review and meta-analysis summarize the data from 32 studies involving a total of 14,515 participants. The results of the meta-analysis shows Zinc supplementation alone causes a significant reduction in LDL-c concentration $(-4.78 \mathrm{mg} / \mathrm{dl}, \mathrm{p}<0.05)$ in the absence of a significant heterogeneity among the studies. Although there is considerable heterogeneity amongst the studies, Zinc supplementation alone reported a statistical significant reduction in serum TC level $(-10.72 \mathrm{mg} / \mathrm{dl}$, $\mathrm{p}<0.05, \mathrm{I} 2=80 \%)$ and TG level $(-8.73 \mathrm{mg} / \mathrm{dl}, \mathrm{p}<0.05$, $\mathrm{I} 2=64 \%)$. When analyzed by health status, Zinc supplementation reported a significant reduction in TC, LDL-c and TG levels in non-healthy patients and the magnitude of reduction was greater than that in overall analysis. In healthy patients there was a minor but significant reduction in TC level whereas changes in LDL-c and 
(I)

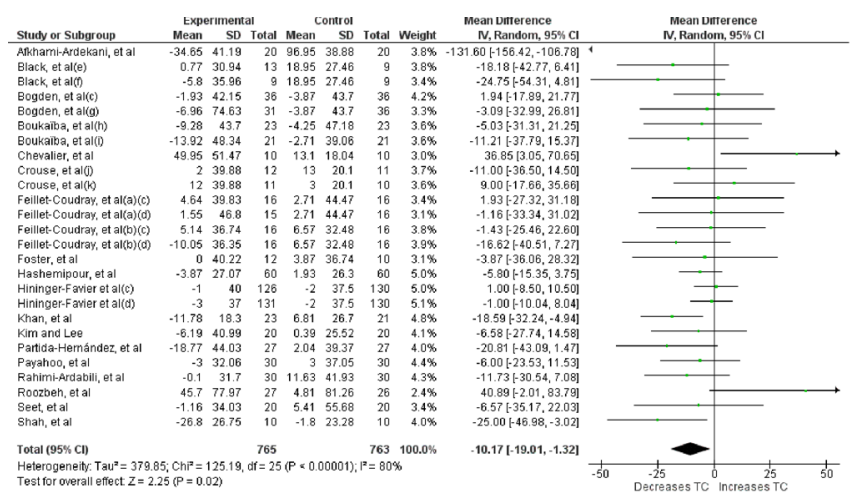

(II)

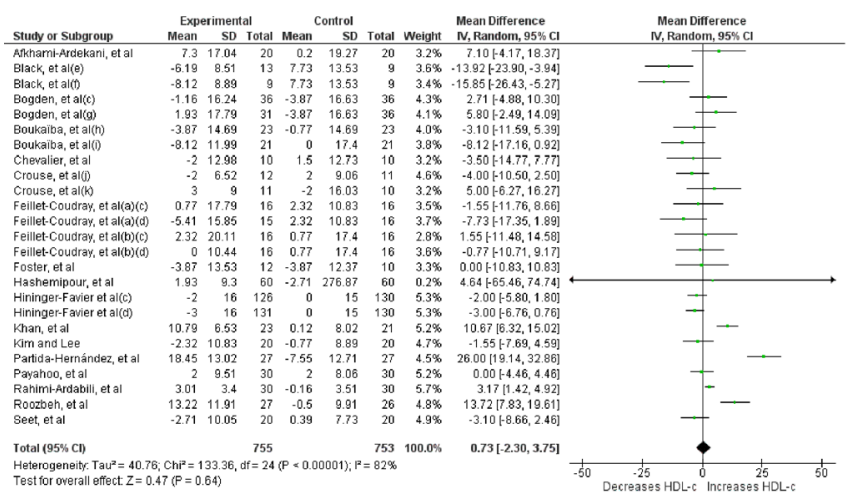

(III)

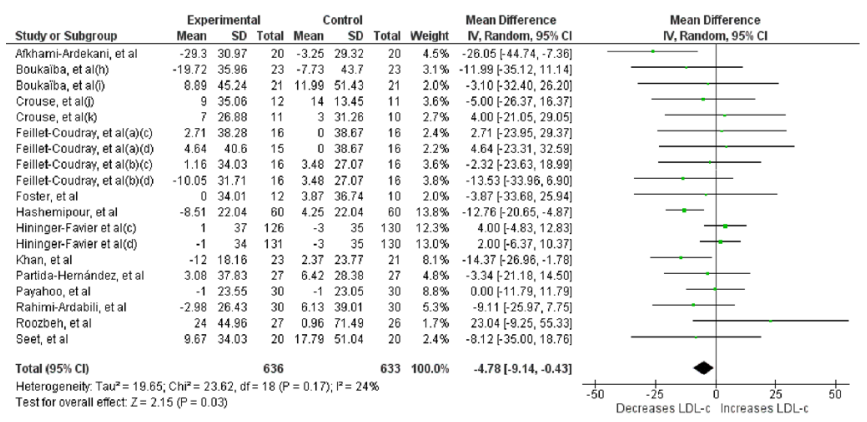

(IV)

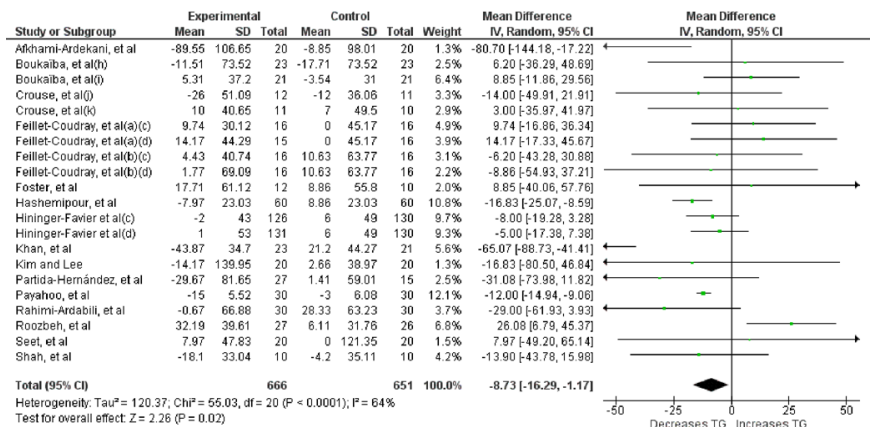

Fig. 3 (See legend on next page.) 
(See figure on previous page.)

Fig. 3 Forest plots showing effect of Zinc supplementation alone (sub-analysis) on; (I) Total cholesterol, (II) HDL cholesterol, (III) LDL cholesterol, IV Triglycerides. (a)- female, (b)- male, (c)- Zinc supplementation 15 mg/day, (d)- Zinc supplementation 30 mg/day, (e)- Zinc supplementation 50 mg/day, (f)- Zinc supplementation 75 mg/day, (g)- Zinc supplementation 100 mg/day, (h)- reference group, (i)- Lean group, (j)- Sedentary males, (k)- Trained males

TG were not significant. When consider HDL-c, Zinc supplementation demonstrated a statistically insignificant increase in the ungrouped analysis whereas a statistically significant increase $(+6.15 \mathrm{mg} / \mathrm{dl})$ among non-healthy patients. These findings are in contrast to results from a previous meta-analysis of randomized controlled trials, where no beneficial effects of Zinc supplementation were observed on plasma TC, LDL-c or TG concentrations in overall, ungrouped analysis or when interventions were grouped by health status [25]. Zinc supplementation has demonstrated a significant increase in HDL-c levels among patients with diabetes mellitus in previous metaanalyses which also supports our finding $[25,56]$. A finding probably resulting from favorable results demonstrated in several studies reported since the time of the previous meta-analysis [17, 21, 28, 33, 42, 46].

Cardiovascular disease is the leading cause of death in much of the modern world and two major underlying causes are disorders of lipid metabolism and metabolic syndrome [57]. Dyslipidemia is the most important risk factor for atherosclerosis [58]. Atherosclerosis is the main aetiological factor behind coronary artery disease, cerebral vascular disease, and peripheral vascular disease [59]. Within the past decade, clinical trials have demonstrated that LDL-c reduction reduce the clinical cardiac events and the arteriographic investigations have demonstrated that LDL-c reduction can significantly reduce the rate of arteriographically defined disease progression [60]. A recent systematic review and meta-regression analysis concluded that simply increasing the level of circulating HDL-c does not reduce the risk of coronary heart disease events, coronary heart disease deaths, or total death and results supported reduction in low density lipoprotein cholesterol as the primary goal for lipid modifying interventions [61]. Each $40 \mathrm{mg} / \mathrm{dl}$ reduction in LDL-c concentration corresponds to $24 \%$ reduction in major cardiovascular events [62]. Therefore, current meta-analysis demonstrates that Zinc supplementation alone can reduce major cardiovascular events by $\sim 2.9 \%$ by lowering LDL-c concentration by $4.78 \mathrm{mg} / \mathrm{dl}$ and by $6.8 \%$ in non-healthy individuals by lowering LDL-c by $11.25 \mathrm{mg} / \mathrm{dl}$ at an average dose of $\sim 40 \mathrm{mg} /$ day. However, atorvastatin a well established drug for hyperlipidaemia has demonstrated $1.8 \mathrm{mmol} / \mathrm{l}$ $(69.6 \mathrm{mg} / \mathrm{dl})$ reduction in LDL-c levels at a dose of $10 \mathrm{mg} /$ day in a meta-analysis involving 164 trials [63].

Previous meta-analyses have reported elevated fasting and non-fasting concentrations of TGs were associated with increased risk of coronary heart disease, even after adjustment for HDL-c concentrations [64, 65]. Furthermore three studies between 2007 and 2008 suggested that raised non-fasting TG was strongly associated with increasing risk of myocardial infarction, ischaemic heart disease, ischaemic stroke and all-cause mortality [66-68]. At mild-to-moderately raised triglyceride concentrations $(2-10 \mathrm{mmol} / \mathrm{l})$, lipoproteins are small enough to enter into arterial wall and thus have the potential to enter into arterial wall and accumulate causing atherosclerosis $[69,70]$. High TG concentrations are a marker for raised remnants rich in cholesterol, which can enter into intima and lead to foam cell formation, atherosclerotic plaques and ultimately cardiovascular disease and increased mortality [71]. Understanding from genetic studies and negative results from randomized trials is low HDL-c might not cause cardiovascular disease as originally thought and this understanding has now generated an interest in elevated levels of TGs [71]. Therefore Zinc supplementation could reduce the cardiovascular events and deaths, as it results in significant reduction in TC, TG and LDL-c.

Dyslipidemia is one of the major risk factors for cardiovascular disease in diabetes mellitus. High plasma TG, increased small dense LDL-c particles, low HDL-c are characteristic features of diabetic dyslipidemia and these lipid changes are mainly attributed to increased free fatty acid flux secondary to insulin resistance [72]. The increase in cardiovascular risk in obesity depends to a significant extent on changes in lipid profile, mainly decreased HDL-c and increased TG and insulin resistance is the central cause for these changes [73]. Prominent and known risk factors that contribute to the increased incidence of atherosclerosis in hemodialysis patients are disorders in lipoprotein metabolism and elevated plasma fibrinogen concentrations [74]. Therefore the participants we categorized as non-healthy (patients with - type 2 diabetes, End stage renal failure and on haemodialysis and obesity) are at increased risk of dyslipidemias. Zinc supplementation significantly reduces TC, LDL-c and TG and elevates HDL-c in non-healthy patients. Elevated plasma concentrations of HDL-c are associated with protection from atherosclerotic cardiovascular disease. Cardio protective effect of HDL-c is due to its role in reverse cholesterol transport in which cholesterol from peripheral tissues is returned to the liver for excretion in the bile, its protective effect on endothelial cells and its antioxidant activity [75]. All these evidence support that Zinc supplementation will effectively reduce the cardiovascular risk among non-healthy patients. 
(I)

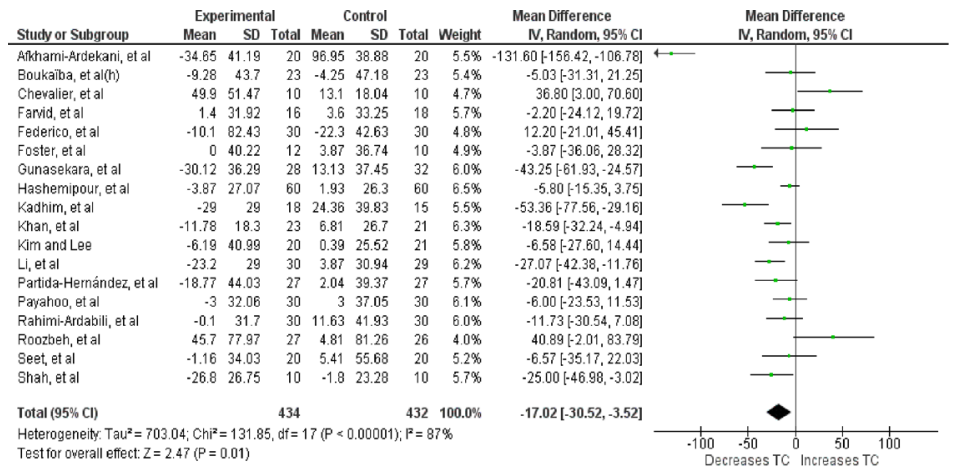

(II)

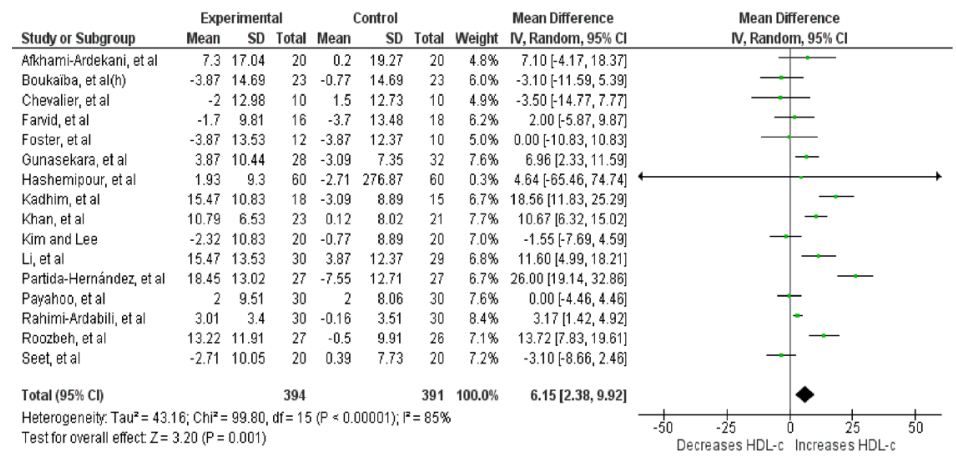

(III)

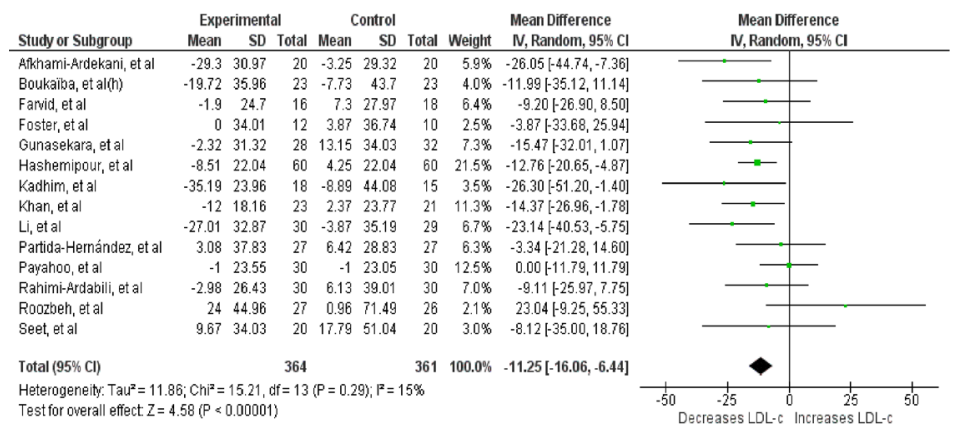

(IV)

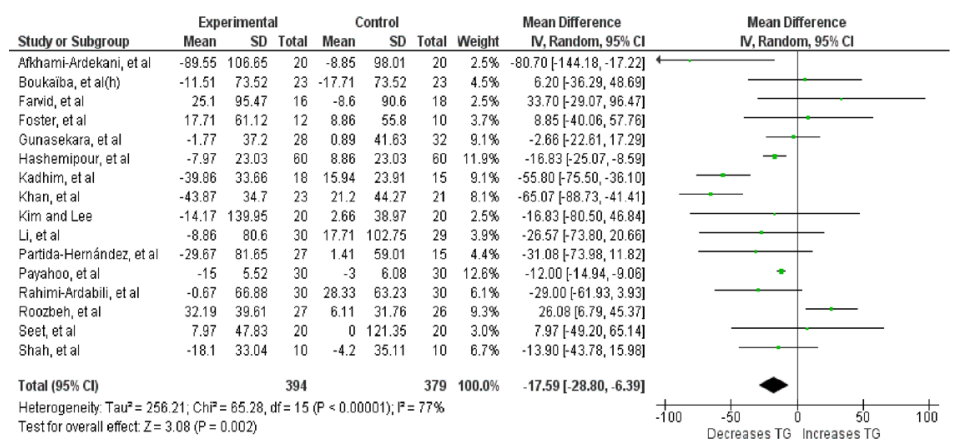

Fig. 4 Forest plots showing effect of Zinc supplementation in non-healthy participants on; (I) Total cholesterol, (II) HDL cholesterol, (III) LDL cholesterol, (IV) Triglycerides 
(I)

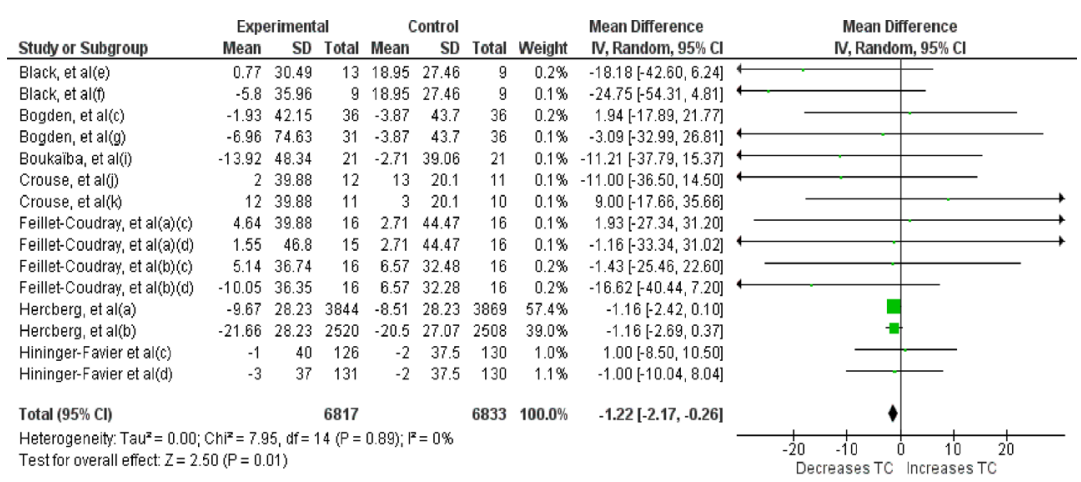

(II)

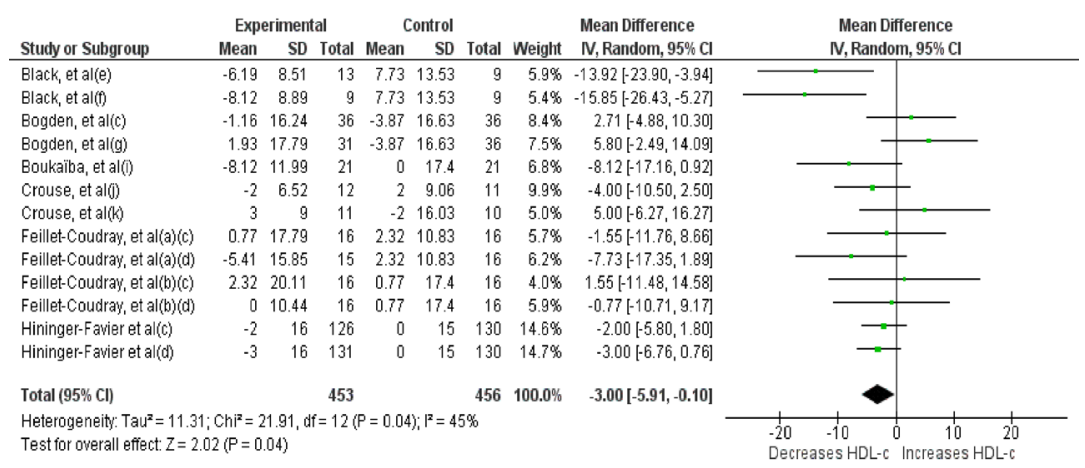

(III)

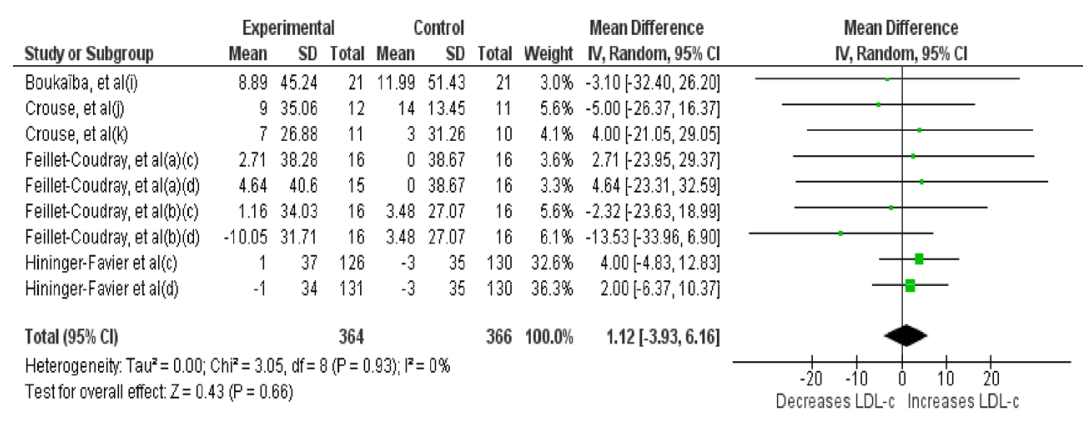

(IV)

\begin{tabular}{|c|c|c|c|c|c|c|c|c|c|}
\hline \multirow[b]{2}{*}{ Study or Subgroup } & \multicolumn{3}{|c|}{ Experimental } & \multicolumn{3}{|c|}{ Control } & \multicolumn{2}{|r|}{ Mean Difference } & \multirow{2}{*}{$\begin{array}{l}\text { Mean Difference } \\
\text { IV, Random, } 95 \% \mathrm{Cl}\end{array}$} \\
\hline & Mean & SD & Total & Mean & SD & Total & Weight & IV, Random, $95 \% \mathrm{Cl}$ & \\
\hline Boukailba, et al(i) & 5.31 & 37.2 & 21 & .3 .54 & 31 & 21 & $10.7 \%$ & $8.85[-11.86,29.56]$ & \\
\hline Crouse, et al(j) & .26 & 51.09 & 12 & -12 & 36.06 & 11 & $3.6 \%$ & $-14.00[-49.91,21.91]$ & \\
\hline Crouse, et al(k) & 10 & 40.65 & 11 & 7 & 49.5 & 10 & $3.0 \%$ & $3.00[-35.97,41.97]$ & \\
\hline Feillet-Coudray, et al(a)(c) & 9.74 & 30.12 & 16 & 0 & 45.17 & 16 & $6.5 \%$ & $9.74[-16.86,36.34]$ & \\
\hline Feillet-Coudray, et al(a)(c) & 14.17 & 44.29 & 15 & 0 & 45.17 & 16 & $4.6 \%$ & $14.17[-17.33,45.67]$ & \\
\hline Feillet-Coudray et al(bi)(c) & 4.43 & 40.74 & 16 & 10.63 & 63.77 & 16 & $3.3 \%$ & $-6.20[-43.28,30.88]$ & \llcorner \\
\hline Feillet-Coudray, et al(b)(c) & 1.17 & 69.09 & 16 & 10.63 & 63.77 & 16 & $2.2 \%$ & $-9.46[-55.53,36.61]$ & $\longmapsto$ \\
\hline Hininger-Favier et al $(c)$ & -2 & 43 & 126 & 6 & 49 & 130 & $36.1 \%$ & $-8.00[-19.28,3.28]$ & \\
\hline Hininger-Favier et al(d) & $i$ & 53 & 131 & 6 & 49 & 130 & $30.0 \%$ & $-5.00[-17.38,7.38]$ & \\
\hline Total $(95 \% \mathrm{Cl})$ & & & 364 & & & 366 & $100.0 \%$ & $.2 .97[-9.75,3.81]$ & \\
\hline $\begin{array}{l}\text { Heterogeneity: Tau }{ }^{2}=0.00 \text {; } \\
\text { Test for overall effect: } z=0 .\end{array}$ & $\begin{array}{l}C^{2}{ }^{2}=4.6 \\
3(P=0 .\end{array}$ & $\begin{array}{l}69, \mathrm{df}= \\
.39)\end{array}$ & & & & & & & $\begin{array}{ccccc}-20 & -10 & 0 & 10 & 2 \\
\text { Decreases TG. Increases TC }\end{array}$ \\
\hline
\end{tabular}

Fig. 5 Forest plots showing effect of Zinc supplementation in healthy participants on; (I) Total cholesterol, (II) HDL cholesterol, (III) LDL cholesterol, (IV) Triglycerides 
Our results demonstrated HDL-c concentration was significantly reduced due to Zinc supplementation among healthy participants. A previous meta-analysis also showed that Zinc supplementation among healthy individuals was associated with a significant reduction in HDL-c concentration supporting our finding [25]. Low HDL-c $(=/<$ $40 \mathrm{mg} / \mathrm{dl}$ ) is one of the 5 major Coronary Heart Disease (CHD) risk factors, and HDL-c level is also a component of the Framingham scoring system, the method used to estimate 10-year CHD risk and determine the intensity of lipid-lowering therapy [75]. Furthermore Zinc supplementation did not demonstrate a significant reduction in LDL-c or in TG despite a minor reduction in TC. Therefore Zinc supplementation may not have much beneficial effects in healthy people.

Several molecular mechanisms are believed to be involved in reduction in serum lipid levels following Zinc supplementation. In Zinc-deficient rats lowered plasma HDL-c and some apoproteins (A1, A2, C and E) but also elevated total cholesterol concentrations have been observed [76, 77]. On the other hand, Zinc supplementation has been shown to inhibit the development of atherosclerosis in rabbits fed a high cholesterol diet [78]. It is well documented that Zinc is an important mediator of insulin storage and secretion from the pancreas [78]. In addition, pancreatic beta-cells utilize a very efficient transporter (ZnT8) to accumulate Zinc inside the cells. Thus, Zinc deficiency or alterations in ZnT8 expression have a potential to depress insulin secretion [79]. Zinc enhances the phosphorylation of insulin-receptor substrates to activate a series of signal transduction, improving insulin sensitivity [80, 81]. Insulin resistance at the adipocytes results in increased release of fatty acids into the circulation and then increased free fatty acid flux to the liver stimulates the assembly and secretion of VLDL resulting in hypertriglyceridemia [82]. Zinc supplementation either improving insulin secretion or reducing insulin resistance as described above inhibits the lipolysis in adipose tissues, reduce free fatty acid release into the circulation and its availability to the liver and excessive lipoprotein synthesis. Besides Zinc contribution to insulin secretion and action, Zinc directly affects lipid metabolism. Recently it has been shown that Zinc deficiency down regulates fatty acid utilization in mitochondria and peroxisomes and up regulates lipid synthesis in the rat liver affecting the expression of genes encoding enzymes contributing to liver lipid homeostasis [83].

The present meta-analysis has notable strengths. These include 1) large number of individuals in the sub group analysis in which the effect of Zinc alone supplementation was studied $(\mathrm{n}=1,528), 2)$ studies were assessed using jaded scale score and the studies with poor methodological quality were excluded from meta-analysis, 3) use of random effect model of meta-analysis which allow heterogeneity among studies and 4) average dose of elemental Zinc used in the interventions included in metaanalysis $(39.3 \mathrm{mg} / \mathrm{d})$ does not exceed the tolerable upper intake level (40 mg elemental Zinc per day in adults) [84]. A limitation of present meta analysis was presence of considerale heterogeneity when assessing the effect of Zinc supplementation on TC anc TG concentrations which stems from; a) Variations in baseline parameters such as serum Zinc status and lipid levels, b) Differences in Zinc doses, formulae, sample sizes and study durations, and c) Limited availability of data on Zinc intake from other sources such as diet.

\section{Conclusions}

The present meta-analysis demonstrates that Zinc supplementation has favourable effects on plasma lipid parameters. Zinc supplementation significantly reduced total cholesterol, LDL cholesterol and triglycerides. In addition to that, Zinc supplementation in non-healthy patients demonstrated a significant elevation of HDL cholesterol. Therefore it may have the potential to reduce the incidence of atherosclerosis related morbidity and mortality especially in non-healthy patients who are at risk of atherosclerosis.

\section{Abbreviations}

FBG: Fasting blood glucose; HbA1c: Glycosylated hemoglobin; HDL-c: High density lipoprotein cholesterol; LDL-c: Low density lipoprotein cholesterol; TG: Triglycerides; TC: total cholesterol; RCT: Randomized control trial; MVM: Multi vitamin mineral.

\section{Competing interests}

The authors declare that they have no competing interests.

\section{Authors' contributions}

PR, RJ, PG, GRC and PK made substantial contribution to conception and study design. PR, WSW and MHI were involved in data collection. PR, WSW and $\mathrm{MHI}$ were involved in refining the study design, statistical analysis and drafting the manuscript. PR, WSW, RJ, PG, PK and GRC critically revised the manuscript. All authors read and approved the final manuscript.

\section{Author details}

'Department of Pharmacology, Faculty of Medicine, University of Colombo, Colombo, Sri Lanka. ${ }^{2}$ Ministry of Health Care and Nutrition, Colombo, Sri Lanka. ${ }^{3}$ Institute of Health and Biomedical Innovation, Queensland University of Technology, Brisbane, Queensland, Australia. ${ }^{4}$ Diabetes Research Unit, Department of Clinical Medicine, Faculty of Medicine, University of Colombo, Colombo, Sri Lanka.

Received: 11 June 2015 Accepted: 24 July 2015

Published online: 04 August 2015

\section{References}

1. King JC, Cousins RJ. Zinc. In: Shils ME, Shike M, Ross AC, Caballero B, Cousins RJ, editors. Modern Nutrition in Health and Disease. Philadelphia: Lippincott Williams and Wilkins; 2006. p. 271-85.

2. Coleman JE. Zinc proteins: enzymes, storage proteins, transcription factors, and replication proteins. Annu Rev Biochem. 1992;61:897-946.

3. Vallee BL, Falchuk KH. The biochemical basis of zinc physiology. Physiol Rev. 1993;73(1):79-118.

4. Chausmer AB. Zinc, insulin and diabetes. J Am Coll Nutr. 1998;17(2):109-15.

5. Pidduck HG, Wren PJ, Evans DA. Hyperzincuria of diabetes mellitus and possible genetical implications of this observation. Diabetes. 1970;19(4):240-7. 
6. Garg VK, Gupta R, Goyal RK. Hypozincemia in diabetes mellitus. J Assoc Physicians India. 1994;42(9):720-1.

7. Kelly F. Use of antioxidants in the prevention and treatment of disease. J Int Fed Clin Chem. 1998;10(1):21-3.

8. World Health Organisation: Quantifying selected major risks to health. In: The World Health Report Geneva; 2002

9. Black RE. Zinc deficiency, infectious disease and mortality in the developing world. J Nutr. 2003;133(5 Suppl 1):1485S-9S.

10. Hambidge M, Krebs NF. Interrelationships of key variables of human zinc homeostasis: relevance to dietary zinc requirements. Annu Rev Nutr. 2001;21:429-52.

11. Prasad AS. Clinical spectrum of human zinc deficiency. In: Prasad AS, editor. Biochemistry of zinc. New York: Plenum Press; 1993. p. 219-58.

12. Beattie JH, Gordon MJ, Rucklidge GJ, Reid MD, Duncan GJ, Horgan GW, et al. Aorta protein networks in marginal and acute zinc deficiency. Proteomics. 2008;8(10):2126-35.

13. Beattie $\mathrm{JH}$, In-Sook K. Is zinc deficiency a risk factor for atherosclerosis? Br J Nutr. 2004;91:177-81.

14. Meerarani P, Ramadass P, Toborek M, Bauer HC, Bauer H, Hennig B. Zinc protects against apoptosis of endothelial cells induced by linoleic acid and tumor necrosis factor alpha. Am J Clin Nutr. 2000;71(1):81-7.

15. Reiterer G, MacDonald R, Browning JD, Morrow J, Matveev SV, Daugherty A, et al. Zinc deficiency increases plasma lipids and atherosclerotic markers in LDL-receptor-deficient mice. J Nutr. 2005;135(9):2114-8.

16. Singh RB, Niaz MA, Rastogi SS, Bajaj S, Gaoli Z, Shoumin Z. Current zinc intake and risk of diabetes and coronary artery disease and factors associated with insulin resistance in rural and urban populations of North India. J Am Coll Nutr. 1998;17(6):564-70.

17. Hashemipour M, Kelishadi R, Shapouri J, Sarrafzadegan N, Amini M, Tavakoli $\mathrm{N}$, et al. Effect of zinc supplementation on insulin resistance and components of the metabolic syndrome in prepubertal obese children. Hormones. 2009:8(4):279-85.

18. Afkhami-Ardekani M, Karimi M, Mohammadi SM, Nourani F. Effect of zinc sulfate supplementation on lipid and glucose in type 2 diabetic patients. Pak J Nutr. 2008;7(4):550-3.

19. Partida-Hernández G, Arreola F, Fenton B, Cabeza M, Román-Ramos R, Revilla-Monsalve MC. Effect of zinc replacement on lipids and lipoproteins in type 2-diabetic patients. Biomed Pharmacother. 2006;60(4):161-8.

20. Kadhim HM, Ismail SH, Hussein KI, Bakir IH, Sahib AS, Khalaf BH, et al. Effects of melatonin and zinc on lipid profile and renal function in type 2 diabetic patients poorly controlled with metformin. J Pineal Res. 2006;41(2):189-93.

21. Kim J, Lee S. Effect of zinc supplementation on insulin resistance and metabolic risk factors in obese Korean women. Nutrition Research and Practice. 2012;6(3):221-5.

22. Roozbeh J, Hedayati P, Sagheb MM, Sharifian M, Jahromi AH, Shaabani S, et al. Effect of zinc supplementation on triglyceride, cholesterol, LDL, and HDL levels in zinc-deficient hemodialysis patients. Ren Fail. 2009;31(9):798-801.

23. Seet RCS, Lee C-YJ, Lim ECH, Quek AML, Huang H, Huang SH, et al. Oral zinc supplementation does not improve oxidative stress or vascular function in patients with type 2 diabetes with normal zinc levels. Atherosclerosis. 2011;219(1):231-9.

24. Freeman SR, Williams HC, Dellavalle RP. The increasing importance of systematic reviews in clinical dermatology research and publication. J Invest Dermatol. 2006;126(11):2357-60.

25. Foster M, Petocz P, Samman S. Effects of zinc on plasma lipoprotein cholesterol concentrations in humans: A meta-analysis of randomised controlled trials. Atherosclerosis. 2010;210(2):344-52.

26. Payahoo L, Ostadrahimi A, Mobasseri M, Bishak YK, Farrin N, Jafarabadi MA, et al. Effects of zinc supplementation on the anthropometric measurements, lipid profiles and fasting blood glucose in the healthy obese adults. Advanced Pharmaceutical Bulletin. 2013;3(1):161-5.

27. Rahimi-Ardabili B, Argani H, Ghorbanihaghjo A, Rashtchizadeh N, Naghavi-Behzad M, Ghorashi S, et al. Paraoxonase enzyme activity is enhanced by zinc supplementation in hemodialysis patients. Ren Fail. 2012;34(9):1123-8.

28. Li Y, Wang C, Zhu K, Feng RN, Sun CH. Effects of multivitamin and mineral supplementation on adiposity, energy expenditure and lipid profiles in obese Chinese women. Int J Obes. 2010;34(6):1070-7.

29. Moher D, Liberati A, Tetzlaff J, Altman DG. Preferred reporting items for systematic reviews and meta-analyses: the PRISMA statement. BMJ. 2009;339:b2535.

30. Cochran WG. The combination of estimates from different experiments. Biometrics. 1954;10:101-29.
31. Higgins IP, Thompson SG. Quantifying heterogeneity in a meta-analysis. Stat Med. 2002;21:1539-58.

32. Rugge B, Balshem H, Sehgal R, Relevo R, Gorman P, Helfand M: Screening and treatment of subclinical hypothyroidism or hyperthyroidism. 2011

33. Foster M, Petocz P, Caterson ID, Samman S: Effects of zinc and a-linolenic acid supplementation on glycemia and lipidemia in women with type 2 diabetes mellitus: a randomized, double-blind, placebo-controlled trial. Journal of Diabetes Research and Clinical Metabolism. 2013;2(1):3.

34. Farvid MS, Siassi F, Jalali M, Hosseini M, Saadat N. The impact of vitamin and/or mineral supplementation on lipid profiles in type 2 diabetes. Diabetes Res Clin Pract. 2004;65(1):21-8.

35. Boukaïba N, Flament C, Acher S, Chappuis P, Piau A, Fusselier M, et al. A physiological amount of zinc supplementation: effects on nutritional, lipid, and thymic status in an elderly population. Am J Clin Nutr. 1993;57(4):566-72.

36. Black MR, Medeiros DM, Brunett E, Welke R. Zinc supplements and serum lipids in young adult white males. Am J Clin Nutr. 1988;47(6):970-5.

37. Bogden JD, Oleske JM, Lavenhar MA, Munves EM, Kemp FW, Bruening KS, et al. Zinc and immunocompetence in elderly people: effects of zinc supplementation for 3 months. Am J Clin Nutr. 1988;48(3):655-63.

38. Chevalier CA, Murphy MD, Suneson J, Vanbeber AD, Gorman MA, Cochran C. The effects of zinc supplementation on serum zinc and cholesterol concentrations in hemodialysis patients. J Ren Nutr. 2002;12(3):183-9.

39. Crouse SF, Hooper PL, Atterbom HA, Papenfuss RL. Zinc ingestion and lipoprotein values in sedentary and endurance-trained men. J Am Med Assoc. 1984;252(6):785-7.

40. Feillet-Coudray C, Meunier N, Bayle D, Brandolini-Bunlon M, Andriollo-Sanchez $\mathrm{M}, \mathrm{O}$ 'Connor JM, et al. Effect of zinc supplementation on in vitro copper-induced oxidation of low-density lipoproteins in healthy French subjects aged 55-70 years: the Zenith Study. The British Journal Of Nutrition. 2006;95(6):1134-42.

41. Hininger-Favier I, Andriollo-Sanchez M, Arnaud J, Meunier N, Bord S, Graham $C$, et al. Age- and sex-dependent effects of long-term zinc supplementation on essential trace element status and lipid metabolism in European subjects: the Zenith Study. The British Journal Of Nutrition. 2007;97(3):569-78.

42. Khan MI, Siddique KU, Ashfaq F, Ali W, Reddy HD, Mishra A. Effect of high-dose zinc supplementation with oral hypoglycemic agents on glycemic control and inflammation in type-2 diabetic nephropathy patients. J Nat Sci Biol Med. 2013:4(2):336-40.

43. Shah DR, Singh PP, Gupta RC, Bhandari TK. Effect of oral zinc sulphate on serum lipids and lipoproteins in human subjects. Indian J Physiol Pharmacol. 1988;32(1):47-50.

44. Hercberg S, Bertrais S, Czernichow S, Noisette N, Galan P, Jaouen A, et al. Alterations of the lipid profile after 7.5 years of low-dose antioxidant supplementation in the SU.VI.MAX study. Lipids. 2005;40(4):335-42.

45. Federico AIP, Federico P, Del Rio A, Mellone MC, Catalano G, Federico P. Effects of selenium and zinc supplementation on nutritional status in patients with cancer of digestive tract. Eur J Clin Nutr. 2001;55:293-7.

46. Gunasekara P, Hettiarachchi M, Liyanage C, Lekamwasam S. Effects of zinc and multimineral vitamin supplementation on glycemic and lipid control in adult diabetes. Diabetes, metabolic syndrome and obesity: targets and therapy. 2011:4:53-60.

47. Jadad AR, Moore RA, Carroll D, Jenkinson C, Reynolds DJ, Gavaghan DJ, et al. Assessing the quality of reports of randomized clinical trials: is blinding necessary? Control Clin Trials. 1996;17(1):1-12.

48. Age-Related Eye Disease Study Research Group. The effect of five-year zinc supplementation on serum zinc, serum cholesterol and hematocrit in persons randomly assigned to treatment group in the age-related eye disease study: AREDS Report No. 7. The Journal Of Nutrition 2002, 132(4):697-702.

49. Brewer GJ, Yuzbasiyan-Gurkan V, Johnson V. Treatment of Wilson's disease with zinc. IX: Response of serum lipids, vol. 118. UNITED STATES: Elsevier; 1991.

50. Freeland-Graves JH, Friedman BJ, Han WH, Shorey RL, Young R. Effect of zinc supplementation on plasma high-density lipoprotein cholesterol and zinc. Am J Clin Nutr. 1982;35(5):988-92.

51. Gatto LM, Samman S. The effect of zinc supplementation on plasma lipids and low-density lipoprotein oxidation in males. Free Radic Biol Med. 1995;19(4):517-21.

52. Hooper PL, Visconti L, Garry PJ, Johnson GE. Zinc lowers high-density lipoprotein-cholesterol levels. JAMA. 1980;244(17):1960-1.

53. Ranga Rao KV, Seshiah V, Kumar TV. Effect of zinc sulfate therapy on control and lipids in type I diabetes. Journal of the Diabetic Association of India. 1990;30(2):38-40. 
54. Samman S, Roberts DC. The effect of zinc supplements on lipoproteins and copper status. Atherosclerosis. 1988;70(3):247-52.

55. Thurnham DI, Munoz N, Lu JB, Wahrendorf J, Zheng SF, Hambidge KM, et al. Nutritional and haematological status of Chinese farmers: the influence of 13.5 months treatment with riboflavin, retinol and zinc. Eur J Clin Nutr. 1988;42(8):647-60.

56. Jayawardena R, Ranasinghe P, Galappatthy P, Malkanthi R, Constantine G, Katulanda P. Effects of zinc supplementation on diabetes mellitus: a systematic review and meta-analysis. Diabetol Metab Syndr. 2012;4(1):13.

57. Russell JC, Proctor SD. Small animal models of cardiovascular disease: tools for the study of the roles of metabolic syndrome, dyslipidemia, and atherosclerosis. Cardiovasc Pathol. 2006;15(6):318-30.

58. Koba S, Hirano T. [Dyslipidemia and atherosclerosis]. Nihon Rinsho. 2011;69(1):138-43.

59. Mallika V, Goswami B, Rajappa M. Atherosclerosis pathophysiology and the role of novel risk factors: A clinicobiochemical perspective. Angiology. 2007:58(5):513-22.

60. Superko HR. Beyond LDL Cholesterol Reduction. Circulation. 1996;94(10):2351-4

61. Briel M, Ferreira-Gonzalez I, You JJ, Karanicolas PJ, Akl EA, Wu P, et al. Association between change in high density lipoprotein cholesterol and cardiovascular disease morbidity and mortality: systematic review and meta-regression analysis. BMJ. 2009;338.

62. Barter P, Gotto AM, LaRosa JC, Maroni J, Szarek M, Grundy SM, et al. HDL Cholesterol, very low levels of LDL cholesterol, and cardiovascular events. N Engl J Med. 2007;357(13):1301-10.

63. Law MR, Wald NJ, Rudnicka AR. Quantifying effect of statins on low density lipoprotein cholesterol, ischaemic heart disease, and stroke: systematic review and meta-analysis. BMJ. 2003;326(7404):7404.

64. Hokanson J, Austin M. Plasma triglyceride is a risk factor for cardiovascular disease independent of HDL cholesterol level; A Meta analysis of population based prospective studies. J Cardiovasc Risk. 1996:3(2):213-9.

65. Sarwar N, Danesh J, Eiriksdottir G, Sigurdsson G, Wareham N, Bingham S, et al. Triglycerides and the risk of coronary heart disease: 10158 incident cases among 262525 participants in 29 Western erospective studies. Circulation. 2007;115(4):450-8.

66. Bansal S, Buring JE, Rifai N, Mora S, Sacks FM, Ridker P. Fasting compared with nonfasting triglycerides and risk of cardiovascular events in women. JAMA. 2007;298(3):309-16.

67. Nordestgaard BG, Benn M, Schnohr P, Tybjærg-Hansen A. NOnfasting triglycerides and risk of myocardial infarction, ischemic heart disease, and death in men and women. JAMA. 2007;298(3):299-308.

68. Freiberg JJ, Tybjærg-Hansen A, Jensen J, Nordestgaard BG. NOnfasting triglycerides and risk of ischemic stroke in the general population. JAMA. 2008;300(18):2142-52.

69. Shaikh M, Wootton R, Nordestgaard BG, Baskerville P, Lumley JS, La Ville AE, et al. Quantitative studies of transfer in vivo of low density, Sf 12-60, and Sf 60-400 lipoproteins between plasma and arterial intima in humans. Arterioscler Thromb Vasc Biol. 1991;11(3):569-77.

70. Nordestgaard BG, Tybjaerg-Hansen A, Lewis B. Influx in vivo of low density, intermediate density, and very low density lipoproteins into aortic intimas of genetically hyperlipidemic rabbits. Roles of plasma concentrations, extent of aortic lesion, and lipoprotein particle size as determinants. Arterioscler Thromb Vasc Biol. 1992;12(1):6-18.

71. Nordestgaard BG, Varbo A. Triglycerides and cardiovascular disease. Lancet. 2014;384(9943):626-35.

72. Mooradian AD. Dyslipidemia in type 2 diabetes mellitus. Nat Clin Pract End Met. 2009;5(3):150-9

73. Franssen R, Monajemi H, Stroes ESG, Kastelein JJP. Obesity and Dyslipidemia. Med Clin North Am. 2011:95(5):893-902.

74. Wanner C, Zimmermann J, Quaschning T, Galle J. Inflammation, dyslipidemia and vascular risk factors in hemodialysis patients. Kidney Int Suppl. 1997;62:S53-55.

75. Assmann G, Gotto AM. HDL Cholesterol and protective factors in atherosclerosis. Circulation. 2004;109(23 suppl 1)):III-8-III-14.

76. Khoja SM, Marzouki ZM, Ashry KM, Hamdi SA. Effect of dietary zinc deficiency on rat lipid concentrations. Saudi Med J. 2002;23(1):82-6.

77. Koo SI, Lee CC. Cholesterol and apolipoprotein distribution in plasma high-density-lipoprotein subclasses from zinc-deficient rats. Am J Clin Nutr. 1989;50(1):73-9.
78. Jenner A, Ren M, Rajendran R, Ning P, Huat BT, Watt F, et al. Zinc supplementation inhibits lipid peroxidation and the development of atherosclerosis in rabbits fed a high cholesterol diet. Free Radic Biol Med. 2007:42(4):559-66.

79. Mocchegiani E, Giacconi R, Malavolta M. Zinc signalling and subcellular distribution: emerging targets in type 2 diabetes. Trends Mol Med. 2008;14(10):419-28.

80. Lynch CJ, Patson BJ, Goodman SA, Trapolsi D, Kimball SR. Zinc stimulates the activity of the insulin- and nutrient-regulated protein kinase mTOR. Am J Physiol Endocrinol Metabvol. 2001;281(1):E25-34.

81. X-h T, Shay NF. Zinc has an insulin-like effect on glucose transport mediated by phosphoinositol-3-kinase and akt in 3 t3-11 fibroblasts and adipocytes. J Nutr. 2001;131(5):1414-20.

82. Ginsberg HN. Insulin resistance and cardiovascular disease. J Clin Invest. 2000;106(4):453-8.

83. Dieck H, Döring F, Fuchs D, Roth H-P, Daniel H. Transcriptome and proteome analysis identifies the pathways that increase hepatic lipid accumulation in Zinc-deficient rats. J Nutr. 2005;135(2):199-205.

84. Saper RB, Rash R. Zinc: An essential micronutrient. Am Fam Physician. 2009;79(9):768

\section{Submit your next manuscript to BioMed Central and take full advantage of:}

- Convenient online submission

- Thorough peer review

- No space constraints or color figure charges

- Immediate publication on acceptance

- Inclusion in PubMed, CAS, Scopus and Google Scholar

- Research which is freely available for redistribution 